\title{
Modeling and Adaptive Coordination Control of a Two-Robot System
}

\author{
Han-Pang Huang and Ruey-Sheng Chen \\ Robotics Laboratory \\ Department of Mechanical Engineering \\ National Taiwan University \\ Taipei, Taiwan 10764, ROC
}

Received September 25, 1990; accepted April 23, 1991

\begin{abstract}
Multiple robots are usually required in a flexible manufacturing system or a complex working environment. In particular, when an object under processing is too big or too heavy, a single robot is insufficient to handle it. Two robots are applicable in such case. This article aims to develop a complete mathematical model and an adaptive controller for two robots carrying a common load. It will be shown that the dynamic model of the two-robot system turns out to be a singular system, taking into account the object dynamics. The condition for which the system model holds is also discussed. The adaptive controller will be used to overcome uncertainties in the object dynamics and robots. The distributed forces in the robot end effectors are determined by an optimal criterion. It will be shown that the adaptive controller surpasses the conventional computed torque controller.
\end{abstract}

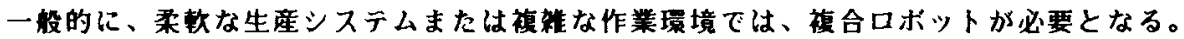
特に、处理対象が大きすきたり重すぎた場合に、単体の口ボットではそれを扱うのに不十 分である。この場合、2台の口ボットを使うのが通当である。この研究の目的は、共通の 負荷を扱う 2 台の口ボットのための完全な数学的モデルと通応型コントローラの開発であ る。2 台のロボット・システムの運動モテルが暴常システムになるのを避けるために、対 象物の運動を考娄していることを示している。また、システム・モテルを保つための条件 についても論じている。対象物の運動とロボットの不確定要素を抑えるために、遥応型コ ントローラが使われている。ロボットのエンドエフェクターに眍分される力は、最逼の基 準によって決定される。道応型コントローラは、従来のトルク計算型のコントローラをし のぐものである。

\section{INTRODUCTION}

Recently, robots have been widely used in industry, space exploration, and medical treatment. However, robot application to sophisticated tasks is quite limited due to the robot capability. A typical example is the two arms of a

Journal of Robotic Systems 9(1), 65-92 (1992).

(C) 1992 by John Wiley \& Sons, Inc.

CCC 0741-2223/92/010065-28\$04.00 
human being. A human being with two arms is able to carry big or heavy objects. Furthermore, they provide a lot of dexterity so that many sophisticated tasks, such as sewing and electronic assembly, can be easily done. In other words, if there are multiple robots or the robot has two arms, the performance and capability of the robot will be greatly enhanced. This motivates researchers to investigate the coordination and control of multiple robots. ${ }^{1-14}$

The cooperation of two robots can be proceeded in three ways: (1) two robots operate in the same workspace without direct contact, such as automobile assembly; (2) two robots form a closed kinematic loop, such as carrying a common load; (3) two robots work on the same object interactively and the relative position, orientation, and reaction forces between two robots are time varying, such as tightening screws. The first problem focuses on the collision avoidance of two robot arms and the task scheduling. The second problem emphasizes the development of two-robot system dynamics, distribution of reaction force, and coordination control. The third problem covers the issues in (1) and (2). Our focus will be on the second problem.

Two robots carrying a common load is particularly useful for the case in which a single robot cannot properly handle the object to be carried. A typical case is that the object to be carried is too big, or too heavy, or both, such that a single robot cannot perform properly. In the past, the research on two-robot systems was focused on the dynamics and control. Dauchey and Zapata, ${ }^{4} \mathrm{Lim}$ and Chyung, ${ }^{5,6}$ and Hayati ${ }^{7}$ use differential motion and homogeneous transformation matrix to first locate the carried objects in Cartesian coordinates and then find the corresponding joint motion. Uchiyama et al. ${ }^{8}$ establish not only the Cartesian coordinates for the carried object but also relative Cartesian location for the two robot end effectors. In their developments of system dynamics, object dynamics are often ignored. Even when object dynamics are considered, they are usually treated as a point mass or the dynamic equations including robot dynamics have to be rederived. ${ }^{7,13}$ When the carried object is big or heavy, the treatment of point mass is not reasonable. On the other hand, rederivation of the complete system dynamics is too time consuming. In those developments, the two-robot system is formulated as a closed-chain system. The closed chain may break as the robot end effector slides off the object. The necessary condition that the closed chain holds is not discussed there. Hence, suitable system dynamics should include the robot dynamics and object dynamics, as given in Huang. ${ }^{9}$ In addition, the condition that the closed chain holds should be addressed. In the control of a two-robot system, the masterslave method or leader-follower method were primarily concerned. ${ }^{10,11,14}$ The master-slave control method has to identify one robot as master, the other as slave. The control is based on the Cartesian coordinate control of the master robot; the slave robot is then controlled through a so-called constrained equation. This type of control method is simple and can be easily extended from single-robot control. However, the target description may not be clear and the carried object may move in zigzag way. Therefore, a fragile object may be torn away. Other researchers employ resolved motion control and the computedtorque method to control the two-robot system. ${ }^{48,15}$ Since the system is highly 
nonlinear and coupling together, those results are not quite satisfactory. Moreover, those control techniques are based on the exact system dynamics, which is not true in general. In particular, the carried object may vary from case to case. Therefore, model uncertainties should be taken into account in the controller design. Although adaptive robot controller has been addressed in the single-robot case, ${ }^{16,17}$ it is seldom discussed in a multiple-robot system.

This article focuses on the development of complete system dynamics and adaptive coordination control of two robots carrying a common load. The system dynamics will take the object dynamics into account. The reaction forces between robot end effectors and the carried object will be regarded as state variables so that the system can be formulated as a singular system. Hence, the reaction force (or contact force) can be directly controlled. To clearly describe the object trajectory and avoid zigzag movement, a nonmaster-slave control method will be used. Furthermore, model uncertainties will be overcome by the proposed adaptive coordination control scheme. The optimal load distribution will be discussed as well. Finally, an example is given to illustrate the above development.

\section{TWO ROBOTS CARRYING A COMMON LOAD}

A schematic diagram of two robots carrying a common load is shown in Figure 1. This robot system is particularly useful for the case in which a single robot cannot properly handle the object to be carried. Multiple robots should be useful for this type of operations. Here, only two robots are considered. It is assumed that the object to be carried is an inertial load. Let $\mathbf{q}^{(1)} \varepsilon \mathbf{R}^{n_{1}}$ and $\mathbf{q}^{(2)} \varepsilon \mathbf{R}^{n_{2}}$ denote the vectors of the first and second robot joint angles, respectively. The equations of motion of each robot without carrying object are given by:

$$
\mathbf{M}_{i}\left(\mathbf{q}^{(i)}\right) \ddot{\mathbf{q}}^{(i)}+\mathbf{F}_{i}\left(\mathbf{q}^{(i)}, \dot{\mathbf{q}}^{(i)}\right)=\boldsymbol{\tau}^{(i)}, \quad i=1,2
$$

where $\mathbf{M}_{i}\left(q^{(i)}\right)$ is the $n_{i} \times n_{i}$ inertial matrix for the $i$ th robot; $\mathbf{F}_{i}\left(\mathbf{q}^{(i)}, \dot{\mathbf{q}}^{(i)}\right)$ is a $n_{i^{-}}$ dimensional vector function containing Coriolis, centrifugal, and gravity terms for the $i$ th robot; and $\tau_{i}$ is an $n_{i}$-dimensional joint torque vector for the $i$ th robot.

To describe the kinematic relations between two robots and object $S$, a coordinate frame diagram is given in Figure 1. Frame $\{0\}$ denotes the world coordinates or Cartesian coordinates. Frames $\{1\}$ and $\{2\}$ are assigned to the base frames. Frames $\{1 e\}$ and $\{2 e\}$ denote the end effector frames of each robot. Frame $\{c\}$ is assigned to the center of mass of the object. Let $\mathbf{p}_{c} \varepsilon \mathbf{R}^{6}$ be the position vector of the center of mass of the object with respect to the world coordinates. Let $\mathbf{B}^{(1)}$ and $\mathbf{B}^{(2)}$ represent the constant $4 \times 4$ homogeneous transformation matrices of frames $\{1\}$ and $\{2\}$ relative to frame $\{0\}$, respectively. $\mathbf{D}^{(1)}$ and $\mathbf{D}^{(2)}$ are $4 \times 4$ transformation matrices of frame $\{c\}$ relative to frames $\{1 e\}$ and $\{2 e\}$, respectively. They are constant matrices since the relative position and orientation between end effectors and the carried object are unchanged during motion. Again, ${ }_{e}^{b} \mathbf{T}^{(i)}$ are transformation matrices of frames $\{i e\}$ relative to frame $\{i\}$, for $i=1,2$. Note that ${ }_{e}^{b} \mathbf{T}^{(1)}$ and ${ }_{e}^{b} \mathbf{T}^{(2)}$ are matrix functions of $\mathbf{q}^{(1)}$ and 


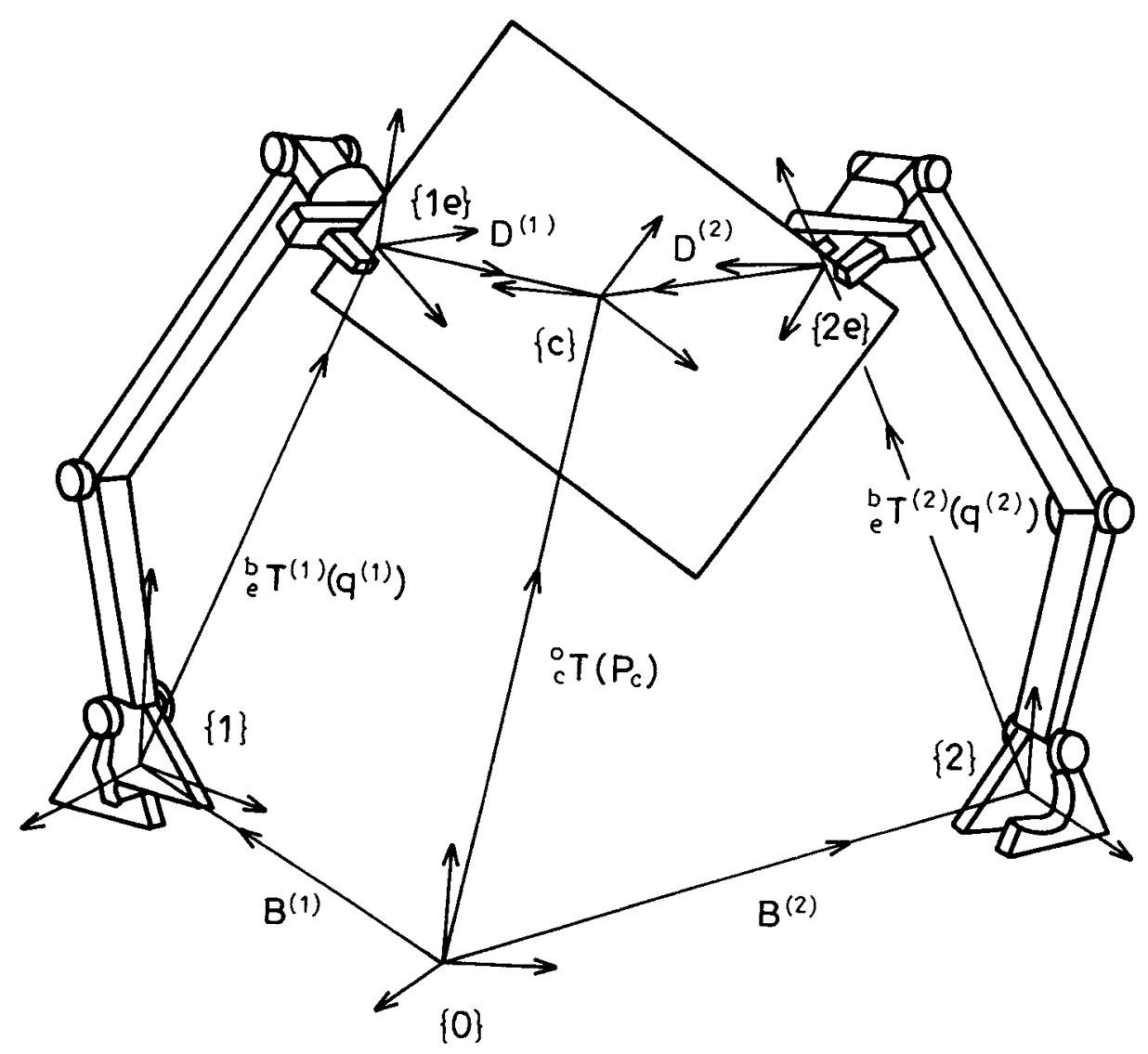

Figure 1. Coordinate frame relation of two-robot system.

$\mathbf{q}^{(2)}$, respectively. ${ }_{c}^{0} \mathbf{T}$ denotes the transformation matrix of $\{c\}$ relative to $\{0\}$. From the coordinate frame diagram, two kinematic relations can be obtained as:

$$
\begin{aligned}
& { }_{c}^{0} \mathbf{T}\left(\mathbf{p}_{c}\right)=\mathbf{B}^{(1) b}{ }_{e} \mathbf{T}^{(1)}\left(\mathbf{q}^{(1)}\right) \mathbf{D}^{(1)} \\
& { }_{c}^{0} \mathbf{T}\left(\mathbf{p}_{c}\right)=\mathbf{B}^{(2) b}{ }_{e}^{\left(\mathbf{T}^{(2)}\right.}\left(\mathbf{q}^{(2)}\right) \mathbf{D}^{(2)}
\end{aligned}
$$

Therefore, the implicit relation between position vector $\mathbf{p}_{c}$ and the first robot joint angle $\mathbf{q}^{(1)}$ can be determined from eq. (2) as:

$$
\mathbf{p}_{c}=\mathbf{H}^{(1)}\left(\mathbf{q}^{(1)}\right)
$$

Similarly, the relation between position vector $\mathbf{p}_{c}$ and the second robot joint angle $\mathbf{q}^{(2)}$ can be determined from eq. (3) as:

$$
\mathbf{p}_{c}=\mathbf{H}^{(2)}\left(\mathbf{q}^{(2)}\right)
$$


Assume the inverse kinematics can be defined as:

$$
\begin{aligned}
\mathbf{q}^{(1)} & =\overline{\mathbf{H}}^{(1)}\left(\mathbf{p}_{c}\right) \\
\mathbf{q}^{(2)} & =\overline{\mathbf{H}}^{(2)}\left(\mathbf{p}_{c}\right)
\end{aligned}
$$

Then, the relevant Jacobian matrices are given as:

$$
\begin{aligned}
\mathbf{J}^{(i)}\left(\mathbf{q}^{(i)}\right) & =\frac{\partial \mathbf{H}^{(i)}}{\partial \mathbf{q}^{(i)}} \\
\overline{\mathbf{J}}^{(i)}\left(\mathbf{p}_{c}\right) & =\frac{\partial \breve{\mathbf{H}}^{(i)}}{\partial \mathbf{p}_{c}}, \quad i=1,2
\end{aligned}
$$

Next, we will derive the dynamic equations of the object. Assume that the end effector of each robot grasps the object at two points, as shown in Figure 2. Let ${ }^{i e} \mathbf{f}^{(i)}$ and ${ }^{i e} \boldsymbol{\tau}^{(i)}$ be the force and moment on the object exerted by the $i$ th robot, $i=1,2$. They are expressed in terms of frames $\{i e\}$, and ${ }^{i} \hat{\mathbf{f}}^{(i)}=\left[{ }^{i e} \mathbf{f}^{(i) T}\right.$ $\left.{ }^{i e} \tau^{(i) r}\right]^{T} . \mathbf{N}^{(1)}$ and $\mathbf{N}^{(2)}$ are the normal force of the robot end effectors, denoted in frames $\{1 e\}$ and $\{2 e\}$, respectively. The mass of the object is $m_{c}$. The center of mass of the object is located at $\left[p_{c x} p_{c y} p_{c z}\right]$, and $\mathbf{p}_{c}=\left[p_{c x}, p_{c y}, p_{c z}, \psi, \theta, \phi\right]^{T}$. The principal moment of inertias with respect to the axes of frame $\{c\}$ are ${ }^{c} \mathbf{I}_{c x x},{ }^{c} \mathbf{I}_{c y y}$,

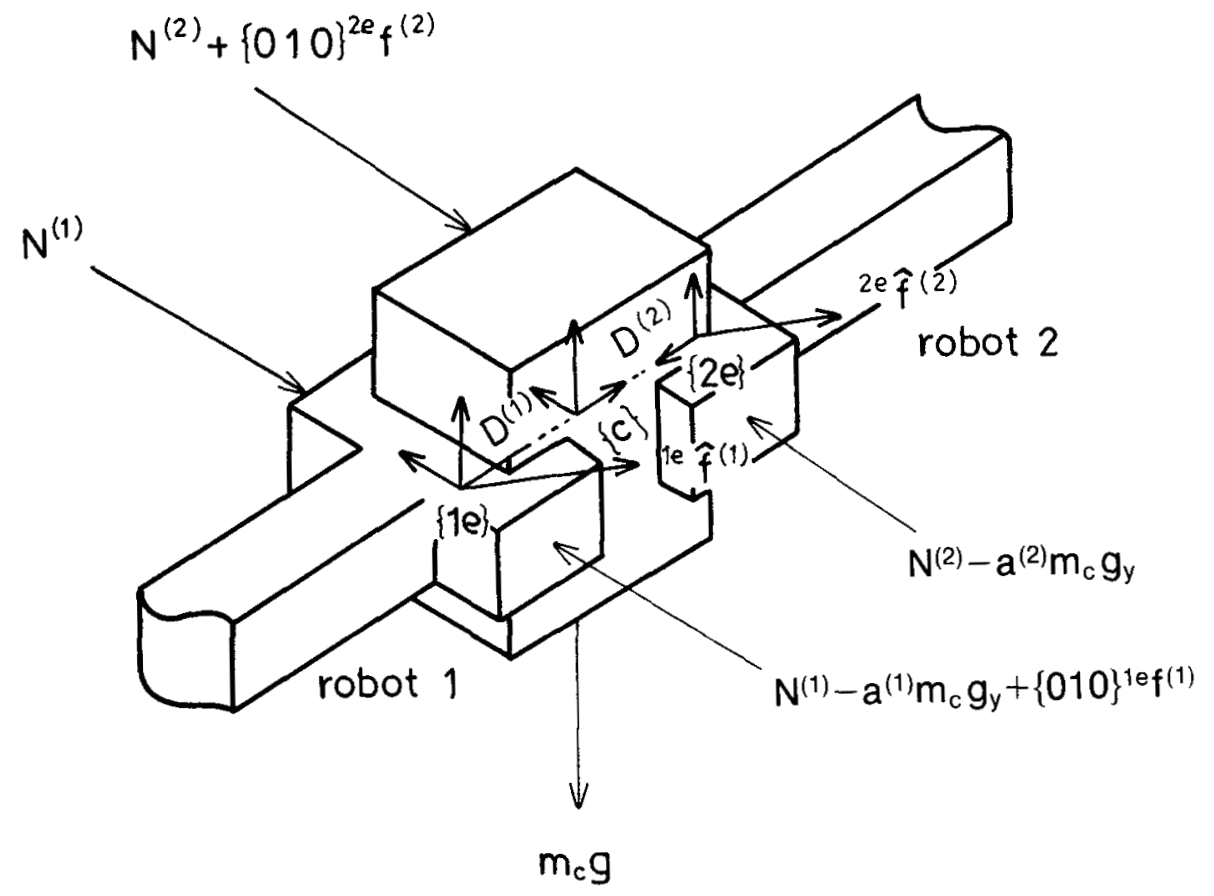

Figure 2. Grasping forces on the object. 
and ${ }^{c} \mathbf{I}_{c z z}$. Assume that the orientation of the object is represented by the ZYX Euler angle. Then, $\left[\begin{array}{ll}\dot{\psi} \dot{\theta} & \dot{\phi}\end{array}\right]$ and $\left[{ }^{c} \omega_{c x}{ }^{c} \omega_{c y}{ }^{c} \omega_{c z}\right]$ can be related by

$$
\begin{aligned}
{\left[\begin{array}{c}
{ }^{c} \omega_{c x} \\
{ }^{c} \omega_{c y} \\
{ }^{c} \omega_{c z}
\end{array}\right] } & =\left[\begin{array}{ccc}
-s \theta & 0 & 1 \\
c \theta s \phi & c \phi & 0 \\
c \theta c \phi & -s \phi & 0
\end{array}\right]\left[\begin{array}{l}
\dot{\psi} \\
\dot{\theta} \\
\dot{\phi}
\end{array}\right] \\
& \triangleq\left[\begin{array}{c}
\dot{\psi} \\
\dot{\theta} \\
\dot{\phi}
\end{array}\right]
\end{aligned}
$$

where $\{g\}$ is the generalized coordinate frame of the ZYX Euler angle. Therefore, the dynamic equations of the object in vector form can be obtained as:

$$
\mathbf{M}_{c}\left(\mathbf{p}_{c}\right) \ddot{\mathbf{p}}_{c}+\mathbf{F}_{c}\left(\mathbf{p}_{c}, \dot{\mathbf{p}}_{c}\right)=\hat{\mathbf{f}}^{(1)}+\hat{\mathbf{f}}^{(2)}
$$

where

$$
\begin{aligned}
& \mathbf{M}_{c}\left(\mathbf{p}_{c}\right)=\left[\begin{array}{cccc}
m_{c} & 0 & 0 & 0 \\
0 & m_{c} & 0 & 0 \\
0 & 0 & m_{c} & 0 \\
0 & 0 & 0 & { }^{\prime} \mathbf{I}_{c x x} s^{2} \theta+{ }^{2} \mathbf{I}_{c y y} c^{2} \theta c^{2} \phi \\
0 & 0 & 0 & { }^{c} \mathbf{I}_{c y y} c \theta s \phi c \phi-{ }^{2} \mathbf{I}_{c z z} c \theta s \phi c \phi \\
0 & 0 & 0 & -{ }^{c} \mathbf{I}_{c x x} s \theta
\end{array}\right] \\
& 0 \\
& 0 \\
& 0 \quad 0 \\
& { }^{c} \mathbf{I}_{c y y} c \theta s \phi c \phi-{ }^{c} \mathbf{I}_{c z z} c \theta s \phi c \phi-{ }^{c} \mathbf{I}_{c x x} s \theta \\
& { }^{c} \mathbf{I}_{c y y} c^{2} \boldsymbol{\phi}+{ }^{c} \mathbf{I}_{c z z} s^{2} \phi \quad 0 \\
& 0 \\
& \mathbf{F}_{c}\left(\mathbf{p}_{c}, \dot{\mathbf{p}}_{c}\right)=\left[\begin{array}{c}
-m_{c} g_{x} \\
-m_{c} g_{y} \\
-m_{c} g_{z} \\
\mathbf{A}(\psi, \theta, \phi, \dot{\psi}, \dot{\theta}, \dot{\phi}) \\
\mathbf{B}(\psi, \theta, \phi, \dot{\psi}, \dot{\theta}, \dot{\phi}) \\
\mathbf{C}(\psi, \theta, \phi, \dot{\psi}, \dot{\theta}, \dot{\phi})
\end{array}\right]
\end{aligned}
$$


and

$$
\begin{aligned}
& \mathbf{A}(\psi, \theta, \phi, \dot{\psi}, \dot{\theta}, \dot{\phi})=-s \theta\left[-{ }^{c} \mathbf{I}_{c x x} c \theta \dot{\theta} \psi+\left({ }^{c} \mathbf{I}_{c z z}-{ }^{c} \mathbf{I}_{c y y}\right)\right. \\
& \quad(\dot{\psi} c \theta c \phi-\dot{\theta} s \phi)]+c \theta s \phi\left\{\mathbf{I}_{c y y}[(-s \theta s \phi \dot{\theta}+c \theta c \phi \dot{\phi}) \psi-s \phi \dot{\phi} \theta]\right. \\
& \left.\quad+\left({ }^{c} \mathbf{I}_{c x x}-{ }^{c} \mathbf{I}_{c z z}\right)(\dot{\phi}-\dot{\psi} s \theta)(\dot{\psi} c \theta c \phi-\dot{\theta} s \phi)\right\} \\
& \quad+c \theta c \phi\left\{\mathbf{I}_{c z z}[(-s \theta c \phi \dot{\theta}-c \theta s \phi \dot{\phi}) \psi-c \phi \dot{\phi} \theta]\right. \\
& \left.\quad+\left({ }^{c} \mathbf{I}_{c y y}-{ }^{c} \mathbf{I}_{c z z}\right)(\dot{\phi}-\dot{\psi} s \theta)(\dot{\psi} c \theta s \phi+\dot{\theta} c \phi)\right\}
\end{aligned}
$$

$\mathbf{B}(\psi, \theta, \phi, \dot{\psi}, \dot{\theta}, \dot{\phi})=c \phi\left\{\mathbf{I}_{c y y}[(-s \theta s \phi \dot{\theta}+c \theta c \phi \dot{\phi}) \psi-s \phi \dot{\phi} \theta]\right.$

$$
\begin{aligned}
& \left.+\left({ }^{c} \mathbf{I}_{c x x}-c \mathbf{I}_{c z z}\right)(\dot{\phi}-\dot{\psi} s \theta)(\dot{\psi} c \theta c \phi-\dot{\theta} s \phi)\right\} \\
& -s \phi\left\{\mathbf{I}_{c z z}[(-s \theta c \phi \dot{\theta}-c \theta s \phi \dot{\phi}) \psi-c \phi \dot{\phi} \theta]\right. \\
& \left.+\left({ }^{c} \mathbf{I}_{c y y}-{ }^{c} \mathbf{I}_{c z z}\right)(\dot{\phi}-\dot{\psi} s \theta)(\dot{\psi} c \theta s \phi+\dot{\theta} c \phi)\right\}
\end{aligned}
$$

$\mathbf{C}(\psi, \theta, \phi, \dot{\psi}, \dot{\theta}, \dot{\phi})=-c \mathbf{I}_{c x x} c \theta \dot{\theta} \psi+\left({ }^{c} \mathbf{I}_{c z z}-{ }^{c} \mathbf{I}_{c y y}\right)(\dot{\psi} c \theta c \phi-\dot{\theta} s \phi)$

$$
c \theta=\cos \theta, s \theta=\sin \theta
$$

and

$$
\begin{aligned}
& \hat{\mathbf{f}}^{(i)}=\left[\begin{array}{c}
\mathbf{f}^{(i)} \\
g \boldsymbol{\tau}^{(i)}
\end{array}\right] \\
& =\left[\begin{array}{cc}
{ }_{i e}^{0} \mathbf{R} & 0 \\
\left({ }_{c}^{g} \mathbf{J}^{T}\right)^{-1}{ }_{i e}^{c} \mathbf{R} K^{(i)} & \left({ }_{c}^{g} \mathbf{J}^{T}\right)^{-1}{ }_{i e}^{c} \mathbf{R}
\end{array}\right]\left[\begin{array}{c}
{ }^{i e} \mathbf{f}^{(i)} \\
{ }^{i e} \boldsymbol{\tau}^{(i)}
\end{array}\right] \\
& =6 \times 1 \text { force vector, } i=1,2
\end{aligned}
$$

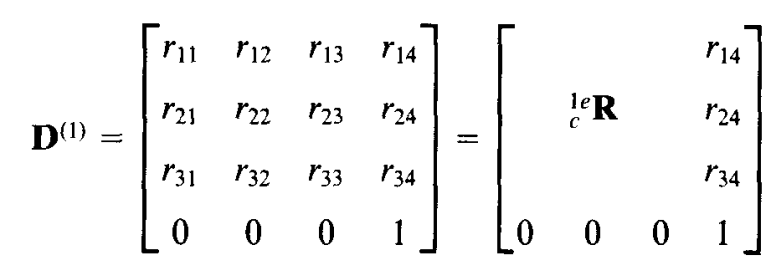

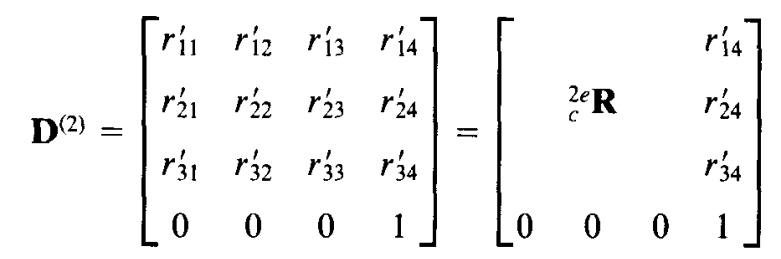

$$
\begin{aligned}
& \mathbf{K}^{(1)}=\left[\begin{array}{ccc}
0 & r_{34} & -r_{24} \\
-r_{34} & 0 & r_{14} \\
r_{24} & -r_{14} & 0
\end{array}\right], \mathbf{K}^{(2)}=\left[\begin{array}{ccc}
0 & r_{34}^{\prime} & -r_{24}^{\prime} \\
-r_{34}^{\prime} & 0 & r_{14}^{\prime} \\
r_{24}^{\prime} & -r_{14}^{\prime} & 0
\end{array}\right]
\end{aligned}
$$


As soon as determining the dynamics of the object, the equations of motion of the two robots, taking into account the reaction forces (and moments) on them, are given by:

$$
\mathbf{M}^{(i)}\left(\mathbf{q}^{(i)}\right) \ddot{\mathbf{q}}^{(i)}+\mathbf{F}^{(i)}\left(\mathbf{q}^{(i)}, \dot{\mathbf{q}}^{(i)}\right)=\mathbf{T}^{(i)}-\mathbf{J}^{(i)}\left(\mathbf{q}^{(i)}\right) \hat{\mathbf{f}}^{(i)}, \quad i=1,2
$$

Thus, the complete equations of motion of the two-robot system, taking into account the object dynamics, are obtained as:

$$
\begin{aligned}
& \mathbf{M}^{(1)}\left(\mathbf{q}^{(1)}\right) \ddot{\mathbf{q}}^{(1)}+\mathbf{F}^{(1)}\left(\mathbf{q}^{(1)}, \dot{\mathbf{q}}^{(1)}\right)=\mathbf{T}^{(1)}-\mathbf{J}^{(1)}\left(\mathbf{q}^{(1)}\right) \hat{\mathbf{f}}^{(1)} \\
& \mathbf{M}^{(2)}\left(\mathbf{q}^{(2)}\right) \ddot{\mathbf{q}}^{(2)}+\mathbf{F}^{(2)}\left(\mathbf{q}^{(2)}, \dot{\mathbf{q}}^{(2)}\right)=\mathbf{T}^{(2)}-\mathbf{J}^{(2)^{T}}\left(\mathbf{q}^{(2)}\right) \hat{\mathbf{f}}^{(2)} \\
& \mathbf{M}_{c}\left(\mathbf{p}_{c}\right) \ddot{\mathbf{p}}_{c}+\mathbf{F}_{c}\left(\mathbf{p}_{c}, \dot{\mathbf{p}}_{c}\right)=\hat{\mathbf{f}}^{(1)}+\hat{\mathbf{f}}^{(2)} \\
& \mathbf{p}_{c}=\mathbf{H}^{(1)}\left(\mathbf{q}^{(1)}\right) \\
& \mathbf{p}_{c}=\mathbf{H}^{(2)}\left(\mathbf{q}^{(2)}\right)
\end{aligned}
$$

Note that eqs. (22) and (23) are equations of constraints rather than kinematic relations of two robots.

The above equations can be conveniently represented in the form of singular system as:

$$
\begin{gathered}
{\left[\begin{array}{ccccc}
\mathbf{M}^{(1)}\left(\mathbf{q}^{(1)}\right) & 0 & 0 & 0 & 0 \\
0 & \mathbf{M}^{(2)}\left(\mathbf{q}^{(2)}\right) & 0 & 0 & 0 \\
0 & 0 & \mathbf{M}_{c}\left(\mathbf{p}_{c}\right) & 0 & 0 \\
0 & 0 & 0 & 0 & 0 \\
0 & 0 & 0 & 0 & 0
\end{array}\right]\left[\begin{array}{c}
\ddot{\mathbf{q}}^{(1)} \\
\ddot{\mathbf{q}}^{(2)} \\
\ddot{\mathbf{p}}_{c} \\
\hat{\mathbf{f}}^{(1)} \\
\dot{\hat{\mathbf{f}}}(2)
\end{array}\right]} \\
=\left[\begin{array}{c}
\mathbf{T}^{(1)}-\mathbf{J}^{(1)}\left(\mathbf{q}^{(1)}\right) \hat{\mathbf{f}}^{(1)}-\mathbf{F}^{(1)}\left(\mathbf{q}^{(1)}, \dot{\mathbf{q}}^{(1)}\right) \\
\mathbf{T}^{(2)}-\mathbf{J}^{(2)}\left(\mathbf{q}^{(2)}\right) \hat{\mathbf{f}}^{(2)}-\mathbf{F}^{(2)}\left(\mathbf{q}^{(2)}, \dot{\mathbf{q}}^{(2)}\right) \\
\hat{\mathbf{f}}^{(1)}+\hat{\mathbf{f}}^{(2)}-\mathbf{F}_{c}\left(\mathbf{p}_{c}, \dot{\mathbf{p}}_{c}\right) \\
\mathbf{p}_{c}-\mathbf{H}^{(1)}\left(\mathbf{q}^{(1)}\right) \\
\mathbf{p}_{c}-\mathbf{H}^{(2)}\left(\mathbf{q}^{(2)}\right)
\end{array}\right]
\end{gathered}
$$

Note that the matrix

$$
\left[\begin{array}{ccccc}
\mathbf{M}^{(1)}\left(\mathbf{q}^{(1)}\right) & 0 & 0 & 0 & 0 \\
0 & \mathbf{M}^{(2)}\left(\mathbf{q}^{(2)}\right) & 0 & 0 & 0 \\
0 & 0 & \mathbf{M}_{c}\left(\mathbf{p}_{c}\right) & 0 & 0 \\
0 & 0 & 0 & 0 & 0 \\
0 & 0 & 0 & 0 & 0
\end{array}\right]
$$


is singular. However, the generalized contact forces $\hat{\mathbf{f}}^{(1)}$ and $\hat{\mathbf{f}}^{(2)}$ can be eliminated by using constraint eqs. (22) and (23). Hence, a nonsingular model of the system is given by:

$$
\mathbf{M}\left(\mathbf{p}_{c}\right) \ddot{\mathbf{p}}_{c}+\mathbf{F}\left(\mathbf{p}_{c}, \dot{\mathbf{p}}_{c}\right)=\mathbf{T}
$$

where

$$
\begin{aligned}
& \mathbf{M}\left(\mathbf{p}_{c}\right)=\mathbf{M}_{c}\left(\mathbf{p}_{c}\right)+\overline{\mathbf{J}}^{(1)}{ }^{T}\left(\mathbf{p}_{c}\right) \mathbf{M}^{(1)}\left[\overline{\mathbf{H}}^{(1)}\left(\mathbf{p}_{c}\right)\right] \overline{\mathbf{J}}^{(1)}\left(\mathbf{p}_{c}\right) \\
& +\overline{\mathbf{J}}^{(2)^{T}}\left(\mathbf{p}_{c}\right) \mathbf{M}^{(2)}\left[\overline{\mathbf{H}}^{(2)}\left(\mathbf{p}_{c}\right)\right] \overline{\mathbf{J}}^{(2)}\left(\mathbf{p}_{c}\right) \\
& \mathbf{F}\left(\mathbf{p}_{c}, \dot{\mathbf{p}}_{c}\right)=\mathbf{F}_{c}\left(\mathbf{p}_{c}, \dot{\mathbf{p}}_{c}\right)+\overline{\mathbf{J}}^{(1)^{T}}\left(\mathbf{p}_{c}\right) \mathbf{F}^{(1)}\left[\overline{\mathbf{H}}^{(1)}\left(\mathbf{p}_{c}\right), \overline{\mathbf{J}}^{(1)}\left(\mathbf{p}_{c}\right) \dot{\mathbf{p}}_{c}\right] \\
& +\overline{\mathbf{J}}^{(2)^{T}}\left(\mathbf{p}_{c}\right) \mathbf{F}^{(2)}\left[\overline{\mathbf{H}}^{(2)}\left(\mathbf{p}_{c}\right), \overline{\mathbf{J}}^{(2)}\left(\mathbf{p}_{c}\right) \dot{\mathbf{p}}_{c}\right] \\
& +\overline{\mathbf{J}}^{(1)^{T}}\left(\mathbf{p}_{c}\right) \mathbf{M}^{(1)}\left[\overline{\mathbf{H}}^{(1)}\left(\mathbf{p}_{c}\right)\right]^{\dot{\overline{\mathbf{J}}}(1)}\left(\mathbf{p}_{c}, \dot{\mathbf{p}}_{c}\right) \dot{\mathbf{p}}_{c} \\
& +\overline{\mathbf{J}}^{(2)}\left(\mathbf{p}_{c}\right) \mathbf{M}^{(2)}\left[\overline{\mathbf{H}}^{(2)}\left(\mathbf{p}_{c}\right)\right] \dot{\overline{\mathbf{J}}}^{(2)}\left(\mathbf{p}_{c}, \dot{\mathbf{p}}_{c}\right) \dot{\mathbf{p}}_{c} \\
& T=\overline{\mathbf{J}}^{(1)}\left(\mathbf{p}_{c}\right) \mathbf{T}^{(1)}+\overline{\mathbf{J}}^{(2)}\left(\mathbf{p}_{c}\right) \mathbf{T}^{(2)}
\end{aligned}
$$

Note that $\mathbf{T}$ depends on both $\mathbf{T}^{(1)}$ and $\mathbf{T}^{(2)}$.

\section{NECESSARY CONDITION FOR CLOSED-CHAIN SYSTEM}

The Cartesian location of the carried object is obtained through kinematic relation. During the development of dynamic equations of the two-robot system, matrices $\mathbf{D}^{(1)}$ and $\mathbf{D}^{(2)}$ are assumed time invariant and configuration independent. Hence, the necessary condition for the kinematic relation (or closed chain) to hold is that the contact between two robot end effectors and carried object must be maintained. Sliding is prohibited. Consider Figure 2 . $\mathbf{N}^{(i)} \mathrm{de}$ notes net grasping force and is perpendicular to $Z-X$ plane in frame $\{i e\} . g_{x}, g_{y}$, and $g_{z}$ represent the gravitational acceleration components in $X, Y$, and $Z$ axes, respectively. Since the object is grasped in the $Y$-direction of frame $\{i e\}$, no sliding occurs in this direction. While in $X-Z$ plane, the sliding is overcome by friction. Suppose $\mu$ is the maximum static friction coefficient between the robot end effector and the carried object; then, the maximum static friction force is:

$$
\mathbf{f}_{\mu}^{(i)}=\mathbf{N}^{(i)} \mu+\left(\mathbf{N}^{(i)}-\mathbf{a}^{(i)} m_{c} g_{y}+\left[\begin{array}{lll}
0 & 1 & 0
\end{array}\right]^{i e} \mathbf{f}^{(i)}\right) \mu, \quad i=1,2
$$

where

$$
\begin{aligned}
& \mathbf{a}^{(1)}=\frac{\left(r_{14}^{\prime 2}+r_{24}^{\prime 2}+r_{34}^{\prime 2}\right)^{1 / 2}}{\left(r_{14}^{2}+r_{24}^{2}+r_{34}^{2}\right)^{1 / 2}+\left(r_{14}^{\prime 2}+r_{24}^{\prime 2}+r_{34}^{\prime 2}\right)^{1 / 2}} \\
& \mathbf{a}^{(2)}=\frac{\left(r_{14}^{2}+r_{24}^{2}+r_{34}^{2}\right)^{1 / 2}}{\left(r_{14}^{2}+r_{24}^{2}+r_{34}^{2}\right)^{1 / 2}+\left(r_{14}^{\prime 2}+r_{24}^{\prime 2}+r_{34}^{\prime 2}\right)^{1 / 2}}
\end{aligned}
$$


Note that $\left[r_{14} r_{24} r_{34}\right]^{T}$ and $\left[r_{14}^{\prime} r_{24}^{\prime} r_{34}^{\prime}\right]$ are position vectors from frame $\{1 e\}$ to $\{c\}$ and frame $\{2 e\}$ to $\{c\}$. To avoid sliding, the following force must be canceled by friction force:

$$
\left.\left\|\mathbf{f}_{x z}^{(i)}\right\|=\left\{\left(\begin{array}{lll}
1 & 0 & 0
\end{array}\right]^{i e} \mathbf{f}^{(i)}-\mathbf{a}^{(i)} m_{c} g_{x}\right)^{2}+\left(\left[\begin{array}{lll}
0 & 0 & 1
\end{array}\right]^{i e} \mathbf{f}^{(i)}-\mathbf{a}^{(i)} m_{c} g_{z}\right)^{2}\right\}^{1 / 2}, \quad i=1,2
$$

Namely,

$$
\mathbf{f}_{\mu}^{(i)} \geq\left\|\mathbf{f}_{x z}^{(i)}\right\|, \quad i=1,2
$$

From eqs. (30)-(33), the necessary condition for the kinematic relation to hold can be obtained in terms of normal contact force $\mathbf{N}^{(i)}$ as:

$$
\begin{aligned}
\mathbf{N}^{(i)} & \geq \frac{-1}{2}\left[\mathbf{a}^{(i)} m_{c} g_{y}+\left[\begin{array}{lll}
0 & 1 & 0
\end{array}\right]{ }^{i e} \mathbf{f}^{(i)}\right]+\frac{1}{2 \mu}\left\{\left(\left[\begin{array}{lll}
1 & 0 & 0
\end{array}\right]^{i e} \mathbf{f}^{(i)}\right.\right. \\
& \left.\left.-\mathbf{a}^{(i)} m_{c} g_{x}\right)^{2}+\left(\left[\begin{array}{lll}
0 & 0 & 1
\end{array}\right]^{i e} \mathbf{f}^{(i)}-\mathbf{a}^{(i)} m_{c} g_{z}\right)^{2}\right\}^{1 / 2}
\end{aligned}
$$

This implies that for sliding avoidance the net normal contact force $\mathbf{N}^{(i)}$ must have a minimum value. In practice, $\mathbf{N}^{(i)}$ also have limitation on the maximum value in order not to damage the carried objects. As to the value of ${ }^{i e} \mathbf{f}^{(i)}$, it can be obtained from force sensor or force estimator.

\section{ADAPTIVE COORDINATION CONTROLLER}

A two-robot system, taking into account the object dynamics, is quite complicated. In the controller design, most researchers simply ignore the object dynamics. However, when two robots carry a common load, the carried object is usually big and heavy. Hence, the object dynamics cannot be ignored. Furthermore, there exist uncertainties of two robots. Due to those uncertainties in the system, if the controller is not "robust" enough, the carried object may be damaged by the robots. To improve the control performance and adapt to varied uncertainties, it is desired to design an adaptive controller for the tworobot system. The adaptive controller will be based on the computed torque control. The optimal grasping force exerted by each robot end effector to the carried object will be designed and controlled. The main idea is to control the center of mass of the carried object to follow a desired trajectory rather than directly control the robot trajectory.

\section{Adaptation Law}

The purpose is to control the center of the mass of the object to track a desired trajectory. Therefore, the system dynamics must be expressed in terms of the object motion, i.e., $\mathbf{p}_{c}, \dot{\mathbf{p}}_{c}$. From eq. (25) we obtain the nonsingular model of the system as:

$$
\mathbf{M}\left(\mathbf{p}_{c}\right) \ddot{\mathbf{p}}_{c}+\mathbf{F}\left(\mathbf{p}_{c}, \dot{\mathbf{p}}_{c}\right)=\mathbf{T}
$$


Note that $\mathbf{M}\left(\mathbf{p}_{c}\right)$ is composed of $\mathbf{M}_{c}, \mathbf{M}^{(1)}\left(\mathbf{q}^{(1)}\right)$, and $\mathbf{M}^{(2)}\left(\mathbf{q}^{(2)}\right)$. $\mathbf{T}$ consists of $\mathbf{T}^{(1)}$ and $\mathbf{T}^{(2)}$. The computed torque control law can be designed as:

$$
\mathbf{T}=\hat{\mathbf{M}}\left(\mathbf{p}_{c}\right)\left[\ddot{\mathbf{p}}_{c d}+\mathbf{K}_{v}\left(\dot{\mathbf{p}}_{c d}-\dot{\mathbf{p}}_{c}\right)+\mathbf{K}_{p}\left(\mathbf{p}_{c d}-\mathbf{p}_{c}\right)\right]+\hat{\mathbf{F}}\left(\mathbf{p}_{c}, \dot{\mathbf{p}}_{c}\right)
$$

where $\mathbf{p}_{c d}, \dot{\mathbf{p}}_{c d}$, and $\ddot{\mathbf{p}}_{c d}$ are the desired motion trajectory of the carried object; $\mathbf{K}_{v}$ and $\mathbf{K}_{p}$ are $6 \times 6$ positive definite matrices, $\mathbf{K}_{v}=\operatorname{diag}\left(k_{v 1}, \ldots, k_{v 6}\right), \mathbf{K}_{p}=$ $\operatorname{diag}\left(k_{p 1}, \ldots, k_{p 6}\right)$; and $\hat{\mathbf{M}}\left(\mathbf{p}_{c}\right)$ and $\hat{\mathbf{F}}\left(\mathbf{p}_{c}, \dot{\mathbf{p}}_{c}\right)$ are the estimated values of $\mathbf{M}\left(\mathbf{p}_{c}\right)$ and $\mathbf{F}\left(\mathbf{p}_{c}, \dot{\mathbf{p}}_{c}\right)$. Using eq. (36) in eq. (35), we obtain the error model:

$$
\ddot{\mathbf{E}}+\mathbf{K}_{\nu} \dot{\mathbf{E}}+\mathbf{K}_{p} \mathbf{E}=\hat{\mathbf{M}}^{-1}\left(\mathbf{p}_{c}\right)\left[\tilde{\mathbf{M}}\left(\mathbf{p}_{c}\right) \ddot{\mathbf{p}}_{c}+\tilde{\mathbf{F}}\left(\mathbf{p}_{c}, \dot{\mathbf{p}}_{c}\right)\right]
$$

where

$$
\begin{gathered}
\mathbf{E}=\mathbf{p}_{c d}-\mathbf{p}_{c} \\
\tilde{\mathbf{M}}\left(\mathbf{p}_{c}\right)=\mathbf{M}\left(\mathbf{p}_{c}\right)-\hat{\mathbf{M}}\left(\mathbf{p}_{c}\right) \\
\tilde{\mathbf{F}}\left(\mathbf{p}_{c}, \dot{\mathbf{p}}_{c}\right)=\mathbf{F}\left(\mathbf{p}_{c}, \dot{\mathbf{p}}_{c}\right)-\hat{\mathbf{F}}\left(\mathbf{p}_{c}, \dot{\mathbf{p}}_{c}\right)
\end{gathered}
$$

It is clear that if the estimated values $\hat{\mathbf{M}}\left(\mathbf{p}_{c}\right)$ and $\hat{\mathbf{F}}\left(\mathbf{p}_{c}, \dot{\mathbf{p}}_{c}\right)$ equal to the actual values $\mathbf{M}\left(\mathbf{p}_{c}\right)$ and $\mathbf{F}\left(\mathbf{p}_{c}, \dot{\mathbf{p}}_{c}\right)$, then eq. (37) reduces to:

$$
\mathbf{M}\left(\mathbf{p}_{c}\right)\left[\ddot{\mathbf{E}}+\mathbf{K}_{v} \dot{\mathbf{E}}+\mathbf{K}_{p} \mathbf{E}\right]=0
$$

Since $\mathbf{M}\left(\mathbf{p}_{c}\right)$ is nonsingular, the error $\mathbf{E}(t)$ will converge to zero. However, it is not true in general. Hence, $\hat{\mathbf{M}}\left(\mathbf{p}_{c}\right)$ and $\hat{\mathbf{F}}\left(\mathbf{p}_{c}, \dot{\mathbf{p}}_{c}\right)$ should be estimated and adjusted. Suppose the overall system has $r$ unknown parameters to be estimated. These unknown parameters are denoted by $\Theta=\left[\Theta_{1}, \ldots, \Theta_{r}\right]^{T}$ and their estimated values by $\hat{\boldsymbol{\Theta}}=\left[\hat{\Theta}_{1}, \ldots, \hat{\Theta}_{r}\right]^{T}$. The parameter error is defined by:

$$
\Phi=\boldsymbol{\Theta}-\hat{\boldsymbol{\Theta}}
$$

Therefore, eq. (37) becomes:

$$
\ddot{\mathbf{E}}+\mathbf{K}_{v} \dot{\mathbf{E}}+\mathbf{K}_{p} \mathbf{E}=\hat{\mathbf{M}}^{-1}\left(\mathbf{p}_{c}\right) \mathbf{W}\left(\mathbf{p}_{c}, \dot{\mathbf{p}}_{c}, \ddot{\mathbf{p}}_{c}\right) \Phi,
$$

where

$$
\mathbf{W}\left(\mathbf{p}_{c}, \dot{\mathbf{p}}_{c}, \ddot{\mathbf{p}}_{c}\right) \Phi=\tilde{\mathbf{M}}\left(\mathbf{p}_{c}\right) \ddot{\mathbf{p}}_{c}+\tilde{\mathbf{F}}\left(\mathbf{p}_{c}, \dot{\mathbf{p}}_{c}\right)
$$

During adaptation phase, the product $\hat{\mathbf{M}}^{-1} \mathbf{W}$ should remain bounded at all times. Since $\mathbf{W}$ consists of bounded functions of the object trajectory, $\mathbf{W}$ will remain bounded as long as the object trajectory remains bounded. The matrix $\hat{\mathbf{M}}\left(\mathbf{p}_{c}\right)$ will be positive definite if all parameters are ensured to remain within a sufficiently small range near the actual parameter value. Namely, the estimates 
of the parameters will be restricted to lie within bounds so that $\hat{\mathbf{M}}$ remains bounded.

The adaptation law will adjust the estimated parameters according to a filtered servo error signal. The filtered servo error $\mathbf{E}_{s}$ is:

$$
\mathbf{E}_{s}=\dot{\mathbf{E}}+\boldsymbol{\alpha} \mathbf{E}
$$

where $\alpha=\operatorname{diag}\left(\alpha_{1}, \ldots, \alpha_{6}\right), \alpha_{i}>0$. Note that the filter need not be implemented, and $\mathbf{E}_{s}$ can be computed from sensor reading. From eq. (43), $\hat{\mathbf{M}}^{-1} \mathbf{W} \Phi$ can be regarded as the input and $\mathbf{E}_{s}$ as the output; then, $\alpha_{i}$ can be chosen so that the transfer function

$$
\frac{\mathbf{E}_{s j}(s)}{\hat{\mathbf{M}} \mathbf{W} \Phi_{j}(s)}=\frac{s+\alpha_{j}}{s^{2}+k_{v j} s+k_{p j}} \quad j=1,6
$$

is strictly positive real. Namely,

$$
0<\alpha_{j}<k_{v j}, \quad j=1,6
$$

The subsequent development is similar to that given by $\mathrm{Craig}^{16}$ for a single robot. The adaptive law can be obtained as:

$$
\hat{\boldsymbol{\Theta}}=\boldsymbol{\Gamma} \mathbf{W}^{T} \hat{\mathbf{M}}^{-1} \mathbf{E}_{s}
$$

where $\Gamma=\operatorname{diag}\left(\Gamma_{1}, \ldots, \Gamma_{r}\right), \Gamma_{i}>0$, is a weighting matrix. To restrict the parameter estimates to lie within bounds, the parameters should be reset to their bounds as the estimates move outside their known bounds. Namely,

$$
\begin{aligned}
& \text { if } \hat{\Theta}_{i}(t) \leq l_{i}-\delta, \quad \text { then } \hat{\Theta}_{i}(t+)=l_{i} \\
& \text { if } \hat{\Theta}_{i}(t) \geq h_{i}+\delta, \quad \text { then } \hat{\Theta}_{i}(t+)=h_{i}
\end{aligned}
$$

where $l_{i}, h_{i}$ are the lower and upper bounds of the parameter estimate $\hat{\boldsymbol{\Theta}}_{i}$ and $\delta$ is a small positive number. At the instant of parameter resetting, the corresponding Lyapunov function used for deriving the adaptation law, eq. (48), should keep negative definite. Craig ${ }^{16}$ has shown that the adaptive scheme is stable in the sense that all signals remain bounded, and trajectory tracking error $\mathbf{E}$ and $\dot{\mathbf{E}}$ converge to zero. In addition, if $\mathbf{W}$ satisfies the persistent excitation, i.e.,

$$
\int_{t_{0}}^{t_{0}+\rho} \mathbf{W}^{T} \mathbf{W d} t \geq \beta \mathbf{I}_{r}, \quad \beta>0, \rho>0
$$

then the parameter error $\Phi$ will converge to zero. In other words, the persistent excitation condition is met if the desired trajectory satisfies

$$
\int_{t_{0}}^{t_{0}+\rho} \mathbf{W}_{d}^{T} \mathbf{W}_{d} \mathrm{~d} t \geq \beta \mathbf{I}_{r}, \quad \beta>0
$$


where $\mathbf{W}_{d}=\mathbf{W}\left(\mathbf{p}_{c d}, \dot{\mathbf{p}}_{c d}, \ddot{\mathbf{p}}_{c d}\right)$. This implies that the parameter convergence can be determined by trajectory planning.

\section{Optimal Grasping Force}

The motion of the carried object is governed by eq. (36), and the parameter update is determined by eq. (48). However, the controller $\mathbf{T}$ in eq. (36) consists of $\mathbf{T}^{(1)}$ and $\mathbf{T}^{(2)}$, namely,

$$
\mathbf{T}=\overline{\mathbf{J}}^{(1)^{T}}\left(\mathbf{p}_{c}\right) \mathbf{T}^{(1)}+\overline{\mathbf{J}}^{(2)}\left(\mathbf{p}_{c}\right) \mathbf{T}^{(2)}
$$

The individual controller of each robot, $\mathbf{T}^{(1)}$ and $\mathbf{T}^{(2)}$, should be further determined.

Let us consider the singular model of the two-robot system, given in eqs. (19)-(23). If the grasping forces, $\hat{\mathbf{f}}^{(1)}$ and $\hat{\mathbf{f}}^{(2)}$, in eqs. (19)-(21) are treated as the control input, then the computed torque controller for the singular model can be obtained as:

$$
\begin{aligned}
\mathbf{T}^{(1)} & -\mathbf{J}^{(1)^{T}}\left(\mathbf{q}^{(1)}\right) \hat{\mathbf{f}}^{(1)}=\hat{\mathbf{M}}^{(1)} \mathbf{J}^{(1)}\left[\mathbf{H}^{(1)}\left(\mathbf{q}^{(1)}\right)\right]\left\{\ddot{\mathbf{p}}_{c d}\right. \\
& \left.+\mathbf{K}_{v}\left[\dot{\mathbf{p}}_{c d}-\mathbf{J}^{(1)}\left(\mathbf{q}^{(1)}\right) \dot{\mathbf{q}}^{(1)}\right]+\mathbf{K}_{p}\left[\mathbf{p}_{c d}-\mathbf{H}^{(1)}\left(\mathbf{q}^{(1)}\right)\right]\right\} \\
& +\hat{\mathbf{M}}^{(1)}\left(\mathbf{q}^{(1)}\right) \dot{\overline{\mathbf{J}}}^{(1)}\left[\mathbf{H}^{(1)}\left(\mathbf{q}^{(1)}\right), \mathbf{J}^{(1)}\left(\mathbf{q}^{(1)}\right) \dot{\mathbf{q}}^{(1)}\right] \mathbf{J}^{(1)}\left(\mathbf{q}^{(1)}\right) \dot{\mathbf{q}}^{(1)} \\
& +\hat{\mathbf{F}}^{(1)}\left(\mathbf{q}^{(1)}, \dot{\mathbf{q}}^{(1)}\right) \\
\mathbf{T}^{(2)} & -\mathbf{J}^{(2)}\left(\mathbf{q}^{(2)}\right) \hat{\mathbf{f}}^{(2)}=\hat{\mathbf{M}}^{(2)} \overline{\mathbf{J}}^{(2)}\left[\mathbf{H}^{(2)}\left(\mathbf{q}^{(2)}\right)\right]\left\{\ddot{\mathbf{p}}_{c d}\right. \\
& \left.+\mathbf{K}_{v}\left[\dot{\mathbf{p}}_{c d}-\mathbf{J}^{(2)}\left(\mathbf{q}^{(2)}\right) \dot{\mathbf{q}}^{(2)}\right]+\mathbf{K}_{p}\left[\mathbf{p}_{c d}-\mathbf{H}^{(2)}\left(\mathbf{q}^{(2)}\right)\right]\right\} \\
& +\hat{\mathbf{M}}^{(2)}\left(\mathbf{q}^{(2)}\right) \dot{\overline{\mathbf{J}}}^{(2)}\left[\mathbf{H}^{(2)}\left(\mathbf{q}^{(2)}\right), \mathbf{J}^{(2)}\left(\mathbf{q}^{(2)}\right) \dot{\mathbf{q}}^{(2)}\right] \mathbf{J}^{(2)}\left(\mathbf{q}^{(2)}\right) \dot{\mathbf{q}}^{(2)} \\
& +\hat{\mathbf{F}}^{(2)}\left(\mathbf{q}^{(2)}, \dot{\mathbf{q}}^{(2)}\right) \\
& \hat{\mathbf{f}}^{(1)}+\hat{\mathbf{f}}^{(2)}=\hat{\mathbf{M}}_{c}\left[\mathbf{H}^{(1)}\left(\mathbf{q}^{(1)}\right)\right]\left\{\ddot{\mathbf{p}}_{c d}+\mathbf{K}_{v}\right. \\
& {\left.\left[\dot{\mathbf{p}}_{c d}-\mathbf{J}^{(1)}\left(\mathbf{q}^{(1)}\right) \dot{\mathbf{q}}^{(1)}\right]+\mathbf{K}_{p}\left[\mathbf{p}_{c d}-\mathbf{H}^{(1)}\left(\mathbf{p}^{(1)}\right)\right]\right\} } \\
& +\hat{\mathbf{F}}_{c}\left[\mathbf{H}^{(1)}\left(\mathbf{q}^{(1)}\right), \mathbf{J}^{(1)}\left(\mathbf{q}^{(1)}\right) \dot{\mathbf{q}}^{(1)}\right]
\end{aligned}
$$

Multiply eq. (52) by $\overline{\mathbf{J}}^{(1)^{T}}\left(\mathbf{p}_{c}\right)$ and eq. (53) by $\overline{\mathbf{J}}^{(2)^{T}}\left(\mathbf{p}_{c}\right)$, then add them together with eq. (54). The resultant controller is exactly the same as the controller $\mathbf{T}$ given in eq. (36). Namely, the individual robot controller $\mathbf{T}^{(1)}$ and $\mathbf{T}^{(2)}$ can be determined from eqs. (52)-(54). Note that relationships $\mathbf{p}_{c}=\mathbf{H}^{(1)}\left(\mathbf{q}^{(1)}\right)=$ $\mathbf{H}^{(2)}\left(\mathbf{q}^{(2)}\right)$ and $\dot{\mathbf{p}}_{c}=\mathbf{J}^{(1)}\left(\mathbf{q}^{(1)}\right) \dot{\mathbf{q}}^{(1)}=\mathbf{J}^{(2)}\left(\dot{\mathbf{q}}^{(2)}\right) \dot{\mathbf{q}}^{(2)}$ have been implicitly used in the above derivations.

From the physical configuration, as shown in Figure 2, the force and moment, ${ }^{1} \hat{\mathbf{f}}^{(1)}$ and ${ }^{2 e} \hat{\mathbf{f}}^{(2)}$, determine the motion of the object; however, there may exist many combinations of ${ }^{1} \hat{\mathbf{f}}^{(1)}$ and ${ }^{2 e} \hat{\mathbf{f}}^{(2)}$. Note that ${ }^{1 e} \hat{\mathbf{f}}^{(i)}=\left[{ }^{i e} \mathbf{f}^{(i) T}{ }^{i e} \boldsymbol{\tau}^{(i) T}\right]^{T}$. Improper combinations of ${ }^{1 e} \hat{\mathbf{f}}^{(1)}$ and ${ }^{2 e} \hat{\mathbf{f}}^{(2)}$ may move the object along the desired trajectory but cause damage on the object. Therefore, the force distribution 
should be carefully designed. To minimize the energy consumption, as well as to avoid damage to the object, the internal reaction force and moment are required to be minimum. The following performance criterion is proposed:

$$
\mathbf{J}={ }^{1 e} \hat{\mathbf{f}}^{(1) T} \mathbf{Q}_{1}{ }^{1 e} \hat{\mathbf{f}}^{(1)}+{ }^{2 e} \hat{\mathbf{f}}^{(2) T} \mathbf{Q}_{2}{ }^{2 e} \hat{\mathbf{f}}^{(2)}
$$

where $\mathbf{Q}_{1}, \mathbf{Q}_{2}$ are weighting matrices. Assume that ${ }_{c}^{g} \mathbf{J}$ is nonsingular, i.e., $\theta \neq \pm 90^{\circ}$ in the Euler angle $(\psi, \theta, \phi)$. Then

$$
i \hat{\mathbf{f}}^{(i)}=\left[\begin{array}{cc}
{ }_{i e}^{0} \mathbf{R} & 0 \\
\left({ }_{c}^{g} \mathbf{J}^{T}\right)^{-1}{ }_{i e}^{c} \mathbf{R} \mathbf{K}^{(i)} & \left({ }_{c}^{g} \mathbf{J}^{T}\right)^{-1}{ }_{i e}^{c} \mathbf{R}
\end{array}\right]^{-1} \hat{\mathbf{f}}^{(i)}, \quad i=1,2
$$

Using eq. (56) in (55), we have:

$$
\mathbf{J}\left(\hat{\mathbf{f}}^{(1)}, \hat{\mathbf{f}}^{(2)}\right)=\left[\begin{array}{l}
\hat{\mathbf{f}}^{(1)} \\
\hat{\mathbf{f}}^{(2)}
\end{array}\right]^{T}\left[\begin{array}{cc}
\mathbf{W}_{1} & 0 \\
0 & \mathbf{W}_{2}
\end{array}\right]\left[\begin{array}{l}
\hat{\mathbf{f}}^{(1)} \\
\hat{\mathbf{f}}^{(2)}
\end{array}\right]
$$

where

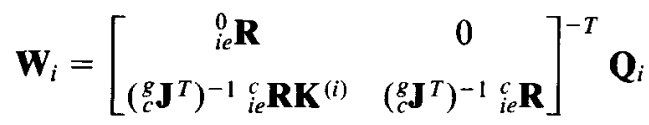

$$
\begin{aligned}
& {\left[\begin{array}{cc}
{ }_{i e}^{0} \mathbf{R} & 0 \\
\left({ }_{c}^{g} \mathbf{J}^{T}\right)^{-1}{ }_{i e}^{c} \mathbf{R} \mathbf{K}^{(i)} & \left({ }_{c}^{g} \mathbf{J}^{T}\right)^{-1}{ }_{i e}^{c} \mathbf{R}
\end{array}\right]^{-1}, \quad i=1,2}
\end{aligned}
$$

From eqs. (57) and (54), the optimal grasping force $\hat{\mathbf{f}}^{(1)}$ and $\hat{\mathbf{f}}^{(2)}$ can be determined as:

$$
\begin{aligned}
& {\left[\begin{array}{l}
\hat{\mathbf{f}}^{(1)} \\
\hat{\mathbf{f}}^{(2)}
\end{array}\right]=\left[\begin{array}{l}
\left(\mathbf{W}_{1}+\mathbf{W}_{2}\right)^{-1} \mathbf{W}_{2}\left\{\hat { \mathbf { M } } _ { c } ( \mathbf { H } ^ { ( 1 ) } ( \mathbf { q } ^ { ( 1 ) } ) ) \left[\ddot{\mathbf{p}}_{c d}+\right.\right. \\
\left(\mathbf{W}_{1}+\mathbf{W}_{2}\right)^{-1} \mathbf{W}_{1}\left\{\hat{\mathbf{M}}_{c}\left(\mathbf{H}^{(2)}\left(\mathbf{q}^{(2)}\right)\right) \ddot{\mathbf{p}}_{c d}+\right.
\end{array}\right.} \\
& \left.\mathbf{K}_{v}\left(\dot{\mathbf{p}}_{c d}-\mathbf{J}^{(1)}\left(\mathbf{q}^{(1)}\right) \dot{\mathbf{q}}^{(1)}\right)+\mathbf{K}_{p}\left(\mathbf{p}_{c d}-\mathbf{H}^{(1)}\left(\mathbf{p}^{(1)}\right)\right)\right] \\
& \left.\mathbf{K}_{v}\left(\dot{\mathbf{p}}_{c d}-\mathbf{J}^{(2)}\left(\mathbf{q}^{(2)}\right) \dot{\mathbf{q}}^{(2)}\right)+\mathbf{K}_{p}\left(\mathbf{p}_{c d}-\mathbf{H}^{(2)}\left(\mathbf{p}^{(2)}\right)\right)\right] \\
& \left.\left.+\hat{\mathbf{F}}_{c}\left(\mathbf{H}^{(1)}\left(\mathbf{q}^{(1)}\right), \mathbf{J}^{(1)}\left(\mathbf{q}^{(1)}\right) \dot{\mathbf{q}}^{(1)}\right)\right\}\right] \\
& \left.\left.+\hat{\mathbf{F}}_{c}\left(\mathbf{H}^{(2)}\left(\mathbf{q}^{(2)}\right), \mathbf{J}^{(2)}\left(\mathbf{q}^{(2)}\right) \dot{\mathbf{q}}^{(2)}\right)\right\}\right]
\end{aligned}
$$

Substituting eq. (59) into eqs. (52) and (53), $\mathbf{T}^{(1)}$ and $\mathbf{T}^{(2)}$ can be obtained as:

$$
\begin{aligned}
& \mathbf{T}^{(1)}=\left[\hat{\mathbf{M}}^{(1)} \overline{\mathbf{J}}^{(1)}+\mathbf{J}^{(1) T}\left(\mathbf{W}_{1}+\mathbf{W}_{2}\right)^{-1} \mathbf{W}_{2} \hat{\mathbf{M}}_{c}\right] \\
& \quad\left[\ddot{\mathbf{p}}_{c d}+\mathbf{K}_{v}\left(\dot{\mathbf{p}}_{c d}-\mathbf{J}^{(1)} \dot{\mathbf{q}}^{(1)}\right)+\mathbf{K}_{p}\left(\mathbf{p}_{c d}-\mathbf{H}^{(1)}\right)\right] \\
& +\hat{\mathbf{M}}^{(1)} \dot{\mathbf{J}}^{(1)} \mathbf{J}^{(1)} \dot{\mathbf{q}}^{(1)}+\hat{\mathbf{F}}^{(1)}+\mathbf{J}^{(1) T}\left(\mathbf{W}_{1}+\mathbf{W}_{2}\right)^{-1} \mathbf{W}_{2} \hat{\mathbf{F}}_{c}
\end{aligned}
$$




$$
\begin{aligned}
& \mathbf{T}^{(2)}=\left[\hat{\mathbf{M}}^{(2)} \overline{\mathbf{J}}^{(2)}+\mathbf{J}^{(2) T}\left(\mathbf{W}_{1}+\mathbf{W}_{2}\right)^{-1} \mathbf{W}_{2} \hat{\mathbf{M}}_{c}\right] \\
& \quad\left[\ddot{\mathbf{p}}_{c d}+\mathbf{K}_{v}\left(\dot{\mathbf{p}}_{c d}-\mathbf{J}^{(2)} \dot{\mathbf{q}}^{(2)}\right)+\mathbf{K}_{p}\left(\mathbf{p}_{c d}-\mathbf{H}^{(2)}\right)\right] \\
& +\hat{\mathbf{M}}^{(2)} \dot{\overline{\mathbf{J}}}^{(2)} \mathbf{J}^{(2)} \dot{\mathbf{q}}^{(2)}+\hat{\mathbf{F}}^{(2)}+\mathbf{J}^{(2) T}\left(\mathbf{W}_{1}+\mathbf{W}_{2}\right)^{-1} \mathbf{W}_{2} \hat{\mathbf{F}}_{c}
\end{aligned}
$$

The arguments in the above are omitted for simplicity. Equations (60) and (61) denote the joint torque controller for each robot. The grasping force exerted by an individual robot can be indirectly controlled through eq. (59). The complete schematic diagram for the adaptive controller is shown in Figure 3.

\section{SIMULATION EXAMPLE}

The above developments have established the system dynamics and the adaptive controller for the two-robot system. In this section, one example will be used to demonstrate the above developments. In the example, two Cartesian robots are used to carry an object from the initial location $(0.4 \mathrm{~m}, 0.2 \mathrm{~m})$ to the final location $(1.2 \mathrm{~m}, 0.6 \mathrm{~m})$. The object is constantly accelerated from the initial location to the middle location $(0.8 \mathrm{~m}, 0.4 \mathrm{~m})$ and then constantly decelerated to the final location. The total time duration is $2.4 \mathrm{~s}$, and the maximum speed is $0.745 \mathrm{~m} / \mathrm{s}$. The schematic diagram is shown in Figure 4 .

First, the dynamic equations of the system can be obtained as follows:

$$
\begin{gathered}
{\left[\begin{array}{cc}
m_{1}+m_{2} & 0 \\
0 & m_{2}
\end{array}\right]\left[\begin{array}{l}
\ddot{\mathbf{q}}_{1}^{(1)} \\
\ddot{\mathbf{q}}_{2}^{(1)}
\end{array}\right]=\left[\begin{array}{l}
\mathbf{T}_{1}^{(1)} \\
\mathbf{T}_{2}^{(1)}
\end{array}\right]-\left[\begin{array}{ll}
0 & 1 \\
1 & 0
\end{array}\right]\left[\begin{array}{l}
\hat{\mathbf{f}}_{1}^{(1)} \\
\hat{\mathbf{f}}_{2}^{(1)}
\end{array}\right]} \\
{\left[\begin{array}{cc}
m_{1}+m_{2} & 0 \\
0 & m_{2}
\end{array}\right]\left[\begin{array}{l}
\ddot{\mathbf{q}}_{1}^{(2)} \\
\ddot{\mathbf{q}}_{2}^{(2)}
\end{array}\right]=\left[\begin{array}{c}
\mathbf{T}_{1}^{(2)} \\
\mathbf{T}_{2}^{(2)}
\end{array}\right]-\left[\begin{array}{ll}
0 & 1 \\
1 & 0
\end{array}\right]\left[\begin{array}{l}
\hat{\mathbf{f}}_{1}^{(2)} \\
\hat{\mathbf{f}}_{2}^{(2)}
\end{array}\right]} \\
{\left[\begin{array}{cc}
m_{c} & 0 \\
0 & m_{c}
\end{array}\right]\left[\begin{array}{l}
\ddot{\mathbf{p}}_{c x} \\
\ddot{\mathbf{p}}_{c y}
\end{array}\right]=\left[\begin{array}{l}
\hat{\mathbf{f}}_{1}^{(1)} \\
\hat{\mathbf{f}}_{2}^{(1)}
\end{array}\right]+\left[\begin{array}{l}
\hat{\mathbf{f}}_{1}^{(2)} \\
\hat{\mathbf{f}}_{2}^{(2)}
\end{array}\right]} \\
{\left[\begin{array}{l}
\mathbf{p}_{c x} \\
\mathbf{p}_{c y}
\end{array}\right]=\left[\begin{array}{c}
\mathbf{q}_{2}^{(1)}+0.1 \\
\mathbf{q}_{1}^{(1)}
\end{array}\right]} \\
{\left[\begin{array}{l}
\mathbf{p}_{c x} \\
\mathbf{p}_{c y}
\end{array}\right]=\left[\begin{array}{c}
1.1-\mathbf{q}_{2}^{(2)} \\
\mathbf{q}_{1}^{(2)}
\end{array}\right]}
\end{gathered}
$$

where $m_{1}, m_{2}, m_{c}$ are masses of link 1 , link2, and the carried object; $\left[\mathbf{q}_{1}^{(1)} \mathbf{q}_{2}^{(1)}\right]^{T}$ is the joint coordinates of robot 1 and $\left[\mathbf{q}_{1}^{(2)} \mathbf{q}_{2}^{(2)}\right]^{T}$ of robot $2 ;\left[\mathbf{p}_{c x} \mathbf{p}_{c y}\right]^{T}$ is the Cartesian coordinates of the center of mass of the carried object; $\left[\mathbf{T}_{1}^{(1)} \mathbf{T}_{2}^{(1)}\right]^{T}$ and $\left[\mathbf{T}_{1}^{(2)} \mathbf{T}_{2}^{(2)}\right]^{T}$ are the generalized force of the two robots; and $\left[\hat{\mathbf{f}}_{1}^{(1)} \hat{\mathbf{f}}_{2}^{(1)}\right]^{T}$ and $\left[\hat{\mathbf{f}}_{1}^{(2)} \hat{\mathbf{f}}_{2}^{(2)}\right]^{T}$ are the grasping forces exerted by robot end effectors to the object. The rele- 


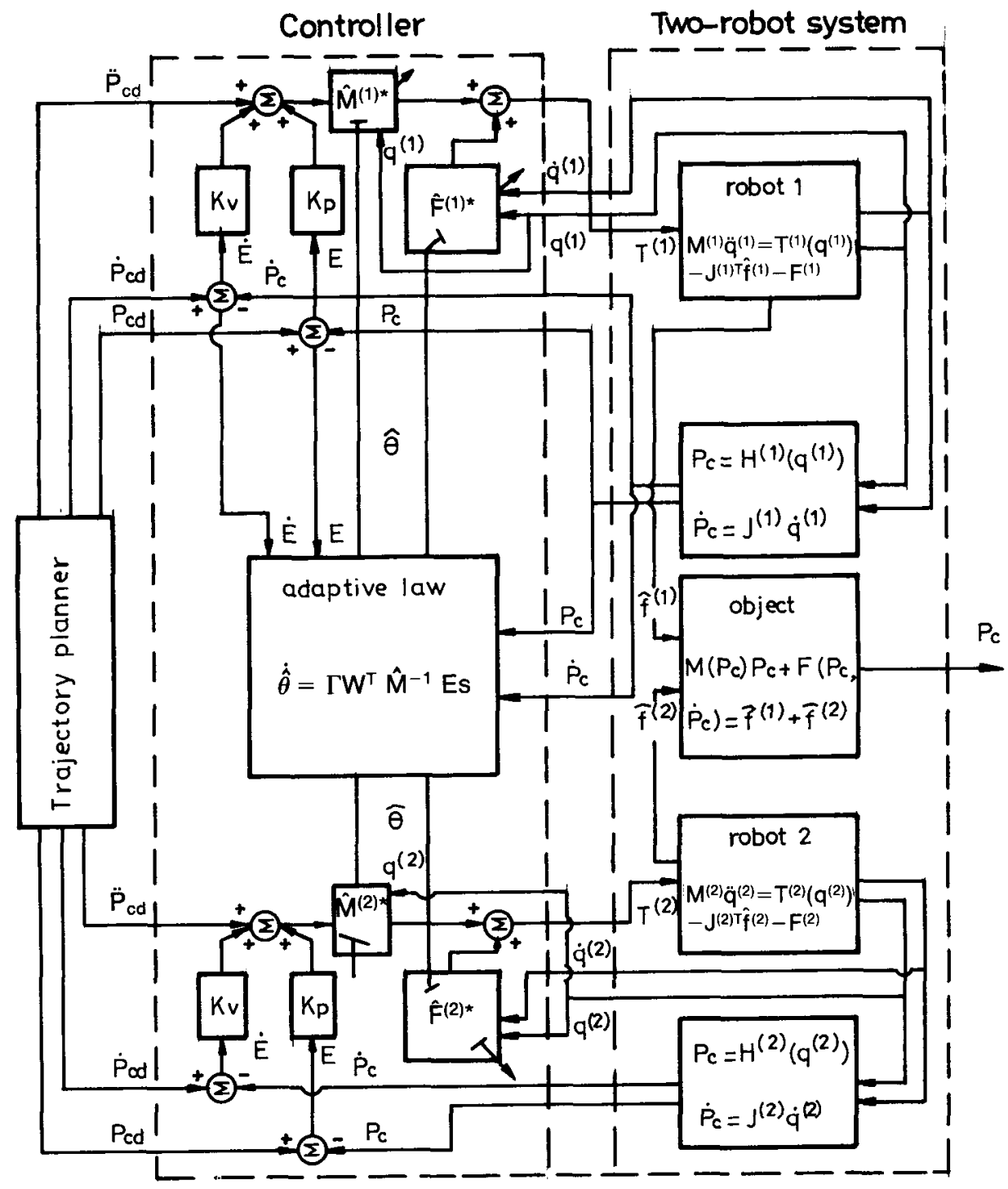

$\hat{\mathrm{M}}^{(1) \star}\left(\mathbf{q}^{(i)}\right)=\left[\hat{\mathrm{M}}^{(i)}\left(\mathbf{q}^{(i)}\right) \bar{J}^{(i)}\left(\mathbf{H}^{(i)}\left(q^{(i)}\right)\right)+J^{(i) \tau}\left(q^{(i)}\right)\left(W_{1}+W_{2}\right)^{-1} W_{2} \hat{M}_{c}\left(H^{(i)}\left(q^{(i)}\right)\right)\right]$

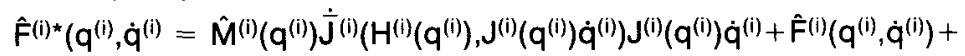
$J^{(i) \tau}\left(q^{(i)}\right)\left(W_{1}+W_{2}\right)^{-1} W_{2} \hat{F}_{c}\left(H^{(i)}\left(q^{(i)}\right), J^{(i)}\left(q^{(i)}\right) \dot{q}^{(i)}\right)$

Figure 3. Schematic diagram of adaptive controller for the two-robot system. 


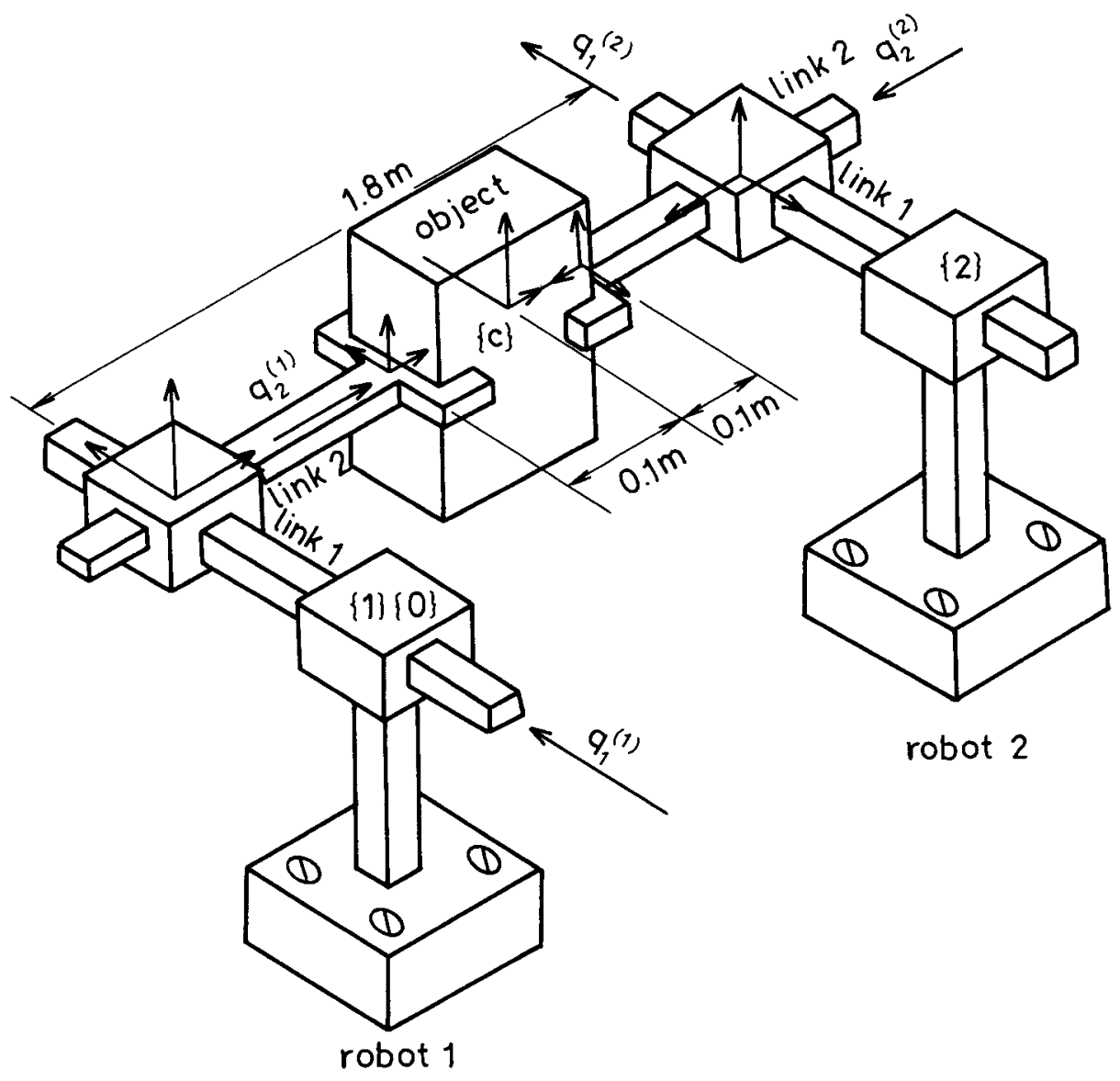

Figure 4. Two robots carrying a common load.

vant data are given in Table I. By eliminating the grasping forces, the system dynamics expressed in nonsingular form can be obtained as:

$$
\begin{gathered}
{\left[\begin{array}{cc}
m_{c}+2 m_{2} & 0 \\
0 & m_{c}+2\left(m_{1}+m_{2}\right)
\end{array}\right]\left[\begin{array}{l}
\ddot{\mathbf{p}}_{c x} \\
\ddot{\mathbf{p}}_{c y}
\end{array}\right]=} \\
{\left[\begin{array}{ll}
0 & 1 \\
1 & 0
\end{array}\right]\left[\begin{array}{l}
\mathbf{T}_{1}^{(1)} \\
\mathbf{T}_{2}^{(1)}
\end{array}\right]+\left[\begin{array}{cc}
0 & -1 \\
1 & 0
\end{array}\right]\left[\begin{array}{l}
\mathbf{T}_{1}^{(2)} \\
\mathbf{T}_{2}^{(2)}
\end{array}\right]}
\end{gathered}
$$

Table I. System parameters of two-robot system.

\begin{tabular}{ccccccc}
\hline $\begin{array}{c}\text { Link 1 } \\
\text { mass } m_{1} \\
(\mathrm{~kg})\end{array}$ & $\begin{array}{c}\text { Link 2 } \\
\text { mass } m_{2} \\
(\mathrm{~kg})\end{array}$ & $\begin{array}{c}\text { Carried } \\
\text { object } m_{c} \\
(\mathrm{~kg})\end{array}$ & $\begin{array}{c}\mathbf{D}_{14}^{(1)} \\
(\mathrm{m})\end{array}$ & $\begin{array}{c}\mathbf{D}_{14}^{(2)} \\
(\mathrm{m})\end{array}$ & $\begin{array}{c}\mathbf{B}_{14}^{(1)} \\
(\mathrm{m})\end{array}$ & $\begin{array}{c}\mathbf{B}_{14}^{(2)} \\
(\mathrm{m})\end{array}$ \\
\hline 5 & 2 & 1 & 0.1 & 0.1 & 0 & 1.8 \\
\hline
\end{tabular}


For the adaptive controller design, the weighting matrices in eq. (55) are chosen as:

$$
\mathbf{Q}_{i}=\left[\begin{array}{ll}
1 & 0 \\
0 & 1
\end{array}\right], \quad i=1,2
$$

From eqs. (60) and (61), the controller can be obtained as:

$$
\begin{gathered}
\mathbf{T}^{(1)}=\left[\begin{array}{c}
\mathbf{T}_{1}^{(1)} \\
\mathbf{T}_{2}^{(1)}
\end{array}\right]=\left[\begin{array}{cc}
0 & \hat{m}_{1}+\hat{m}_{2}+\frac{\hat{m}_{c}}{2} \\
\hat{m}_{2}+\frac{\hat{m}_{c}}{2} & 0
\end{array}\right] \\
{\left[\begin{array}{c}
\ddot{\mathbf{p}}_{c x d}+\mathbf{K}_{v 1}\left(\dot{\mathbf{p}}_{c x d}-\dot{\mathbf{q}}_{2}^{(1)}\right)+\mathbf{K}_{p 1}\left[\mathbf{p}_{c x d}-\left(\mathbf{q}_{2}^{(1)}+0.1\right)\right] \\
\ddot{\mathbf{p}}_{c y d}+\mathbf{K}_{v 2}\left(\dot{\mathbf{p}}_{c y d}-\dot{\mathbf{q}}_{1}^{(1)}\right)+\mathbf{K}_{p 2}\left(\mathbf{p}_{c y d}-\mathbf{q}_{1}^{(1)}\right)
\end{array}\right]} \\
\mathbf{T}^{(2)}=\left[\begin{array}{c}
\mathbf{T}_{1}^{(2)} \\
\mathbf{T}_{2}^{(2)}
\end{array}\right]=\left[\begin{array}{cc}
0 & \hat{m}_{1}+\hat{m}_{2}+\frac{\hat{m}_{c}}{2} \\
-\hat{m}_{2}-\frac{\hat{m}_{c}}{2} & 0
\end{array}\right] \\
{\left[\begin{array}{c}
\ddot{\mathbf{p}}_{c x d}+\mathbf{K}_{v 1}\left(\dot{\mathbf{p}}_{c x d}-\dot{\mathbf{q}}_{2}^{(2)}\right)+\mathbf{K}_{p 1}\left[\mathbf{p}_{c x d}-\left(1.1-\mathbf{q}_{2}^{(2)}\right)\right] \\
\ddot{\mathbf{p}}_{c y d}+\mathbf{K}_{v 2}\left(\dot{\mathbf{p}}_{c y d}-\dot{\mathbf{q}}_{1}^{(2)}\right)+\mathbf{K}_{p 2}\left(\mathbf{p}_{c y d}-\mathbf{q}_{1}^{(2)}\right)
\end{array}\right]}
\end{gathered}
$$

where $\hat{m}_{1}, \hat{m}_{2}, \hat{m}_{c}$ are the estimate values of $m_{1}, m_{2}, m_{c}$ and $\left[\mathbf{p}_{c x d} \mathbf{p}_{c y d}\right]^{T}$ is the desired trajectory. From eq. (48), the adaptive law is given by:

$$
\begin{aligned}
& \dot{\hat{\mathbf{\Theta}}}_{1}=\boldsymbol{\Gamma}_{1} \ddot{\mathbf{q}}_{2}^{(1)}\left[\left(\dot{\mathbf{p}}_{c x d}-\dot{\mathbf{q}}_{2}^{(1)}\right)+\alpha_{1}\left(\mathbf{p}_{c x d}-\left(\mathbf{q}_{2}^{(1)}+0.1\right)\right)\right] / \hat{\boldsymbol{\Theta}}_{1} \\
& \hat{\mathbf{\Theta}}_{2}=\boldsymbol{\Gamma}_{2} \ddot{\mathbf{q}}_{1}^{(1)}\left[\left(\dot{\mathbf{p}}_{c y d}-\dot{\mathbf{q}}_{1}^{(1)}\right)+\alpha_{2}\left(\mathbf{p}_{c y d}-\mathbf{q}_{1}^{(1)}\right)\right] / \hat{\boldsymbol{\Theta}}_{1}
\end{aligned}
$$

where $\hat{\boldsymbol{\Theta}}_{1}=\hat{m}_{c}+2 \hat{m}_{2}$ and $\hat{\boldsymbol{\Theta}}_{2}=\hat{m}_{c}+2\left(\hat{m}_{1}+\hat{m}_{2}\right)$. In the adaptive law, $\boldsymbol{\Theta}_{1} \neq 0$ and $\boldsymbol{\Theta}_{2} \neq 0$. If quantities $\ddot{\mathbf{q}}_{1}^{(1)}, \ddot{\mathbf{q}}_{2}^{(1)}$ cannot be measured, they can be calculated by the following equation:

$$
\left.\ddot{\mathbf{q}}_{i}^{(i)}[(\mathbf{k}+1) \Delta]-\dot{\mathbf{q}}_{i}^{(i)}(\mathbf{k} \Delta)\right] / \Delta, \quad i=1 \text { or } 2
$$

where $\Delta$ is the servo sampling time. Note that the parameter estimates will converge since the persistent excitation

$$
\begin{gathered}
\int_{t_{0}}^{t_{0}+\rho} \mathbf{W}_{d}^{T} \mathbf{W}_{d} \mathrm{~d} t=\int_{t_{0}}^{t_{0}+\rho}\left[\begin{array}{cc}
0.5556 & 0 \\
0 & 0.2778
\end{array}\right] \\
{\left[\begin{array}{cc}
0.5556 & 0 \\
0 & 0.2778
\end{array}\right] \mathrm{d} t \geq \alpha\left[\begin{array}{ll}
1 & 0 \\
0 & 1
\end{array}\right], \quad \rho, \alpha>0}
\end{gathered}
$$


Table II. Parameters for case 1.

\begin{tabular}{cccccccc}
\hline $\boldsymbol{\Gamma}_{1}$ & $\boldsymbol{\Gamma}_{2}$ & $\alpha_{1}$ & $\alpha_{2}$ & $\mathbf{K} v_{1}$ & $\mathbf{K} v_{2}$ & $\mathbf{K} p_{1}$ & $\mathbf{K} p_{2}$ \\
\hline 10,000 & 50,000 & 1 & 1 & 10 & 10 & 25 & 25 \\
\hline
\end{tabular}

is satisfied. With this adaptive law, the adaptive controller can be obtained as:

$$
\begin{aligned}
& \mathbf{T}^{(1)}= {\left[\begin{array}{c}
\frac{\hat{\boldsymbol{\Theta}}_{2}}{2}\left[\ddot{\mathbf{p}}_{c y d}+\mathbf{K}_{v 2}\left(\dot{\mathbf{p}}_{c y d}-\dot{\mathbf{q}}_{1}^{(1)}\right)+\mathbf{K}_{p 2}\left(\mathbf{p}_{c y d}-\mathbf{q}_{1}^{(1)}\right)\right] \\
\frac{\hat{\boldsymbol{\Theta}}_{1}}{2}\left[\ddot{\mathbf{p}}_{c x d}+\mathbf{K}_{v 1}\left(\dot{\mathbf{p}}_{c x d}-\dot{\mathbf{q}}_{2}^{(1)}\right)+\mathbf{K}_{p 1}\left(\mathbf{p}_{c x d}-\left(\mathbf{q}_{2}^{(1)}+0.1\right)\right)\right]
\end{array}\right] } \\
& \mathbf{T}^{(2)}=\left[\begin{array}{c}
\frac{\hat{\boldsymbol{\Theta}}_{2}}{2}\left[\ddot{\mathbf{p}}_{c y d}+\mathbf{K}_{v 2}\left(\dot{\mathbf{p}}_{c y d}-\dot{\mathbf{q}}_{1}^{(2)}\right)+\mathbf{K}_{p 2}\left(\mathbf{p}_{c y d}-\mathbf{q}_{1}^{(2)}\right)\right] \\
\frac{-\hat{\boldsymbol{\Theta}}_{1}}{2}\left\{\ddot{\mathbf{p}}_{c x d}+\mathbf{K}_{v 1}\left(\dot{\mathbf{p}}_{c x d}-\dot{\mathbf{q}}_{2}^{(2)}\right)+\mathbf{K}_{p 1}\left[\mathbf{p}_{c x d}-\left(1.1-\mathbf{q}_{2}^{(1)}\right)\right]\right\}
\end{array}\right]
\end{aligned}
$$

Two cases will be simulated. One concerns the parameters' convergence; the other considers the sudden change of the payload.

\section{Case 1}

This case deals with the parameter convergence. Inexact initial system parameters are used to test the performance of the adaptive controller. Results are also compared with those by using traditional computed torque controllers. True parameters are $\left[\boldsymbol{\Theta}_{1} \boldsymbol{\Theta}_{2}\right]^{T}=\left[\begin{array}{ll}5 & 15\end{array}\right]^{T}$. The initial estimates are $\left[\hat{\boldsymbol{\Theta}}_{1}(0) \hat{\boldsymbol{\Theta}}_{2}(\mathbf{0})\right]^{T}$ $=\left[\begin{array}{ll}8 & 20\end{array}\right]^{T}$. The other relevant parameters are given in Table II. The results are shown in Figures 5-11. Clearly, the adaptive controller gives better performance than the computed torque controller. It seems that the parameters will converge to their true values in spite of inexact initial estimates. In other simulation runs, it was found that larger weighting factors $\boldsymbol{\Gamma}_{1}, \boldsymbol{\Gamma}_{2}$ or larger filter gains $\alpha_{1}, \alpha_{2}$ result in faster convergence and better control performance.

\section{Case 2}

This case deals with the sudden change of the parameters. At $t=0.64 \mathrm{~s}$, one additional payload with the mass of $1 \mathrm{~kg}$ is added to the system. The other relevant parameters are given in Table III. The simulation results are given in Figures 12-18. Again, the adaptive controller surpasses the performance of the

Table III. Parameters for case 2.

\begin{tabular}{cccccccccc}
\hline$\hat{\boldsymbol{\Theta}}_{1}(0)$ & $\hat{\boldsymbol{\Theta}}_{2}(0)$ & $\boldsymbol{\Gamma}_{1}$ & $\boldsymbol{\Gamma}_{2}$ & $\alpha_{\mathrm{a}}$ & $\alpha_{2}$ & $\mathbf{K} v_{1}$ & $\mathbf{K} v_{2}$ & $\mathbf{K} p_{1}$ & $\mathbf{K} p_{2}$ \\
\hline 5 & 15 & 10,000 & 50,000 & 1 & 1 & 10 & 10 & 25 & 25 \\
\hline
\end{tabular}




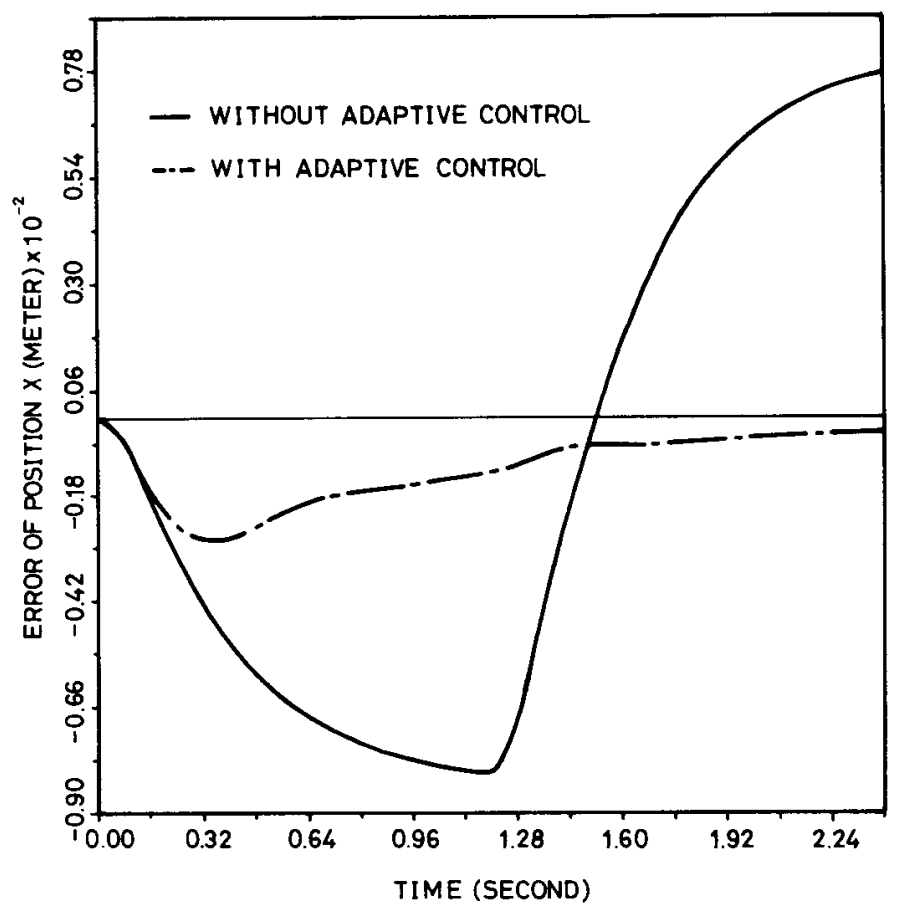

Figure 5. Case 1, position error in $x$-axis.

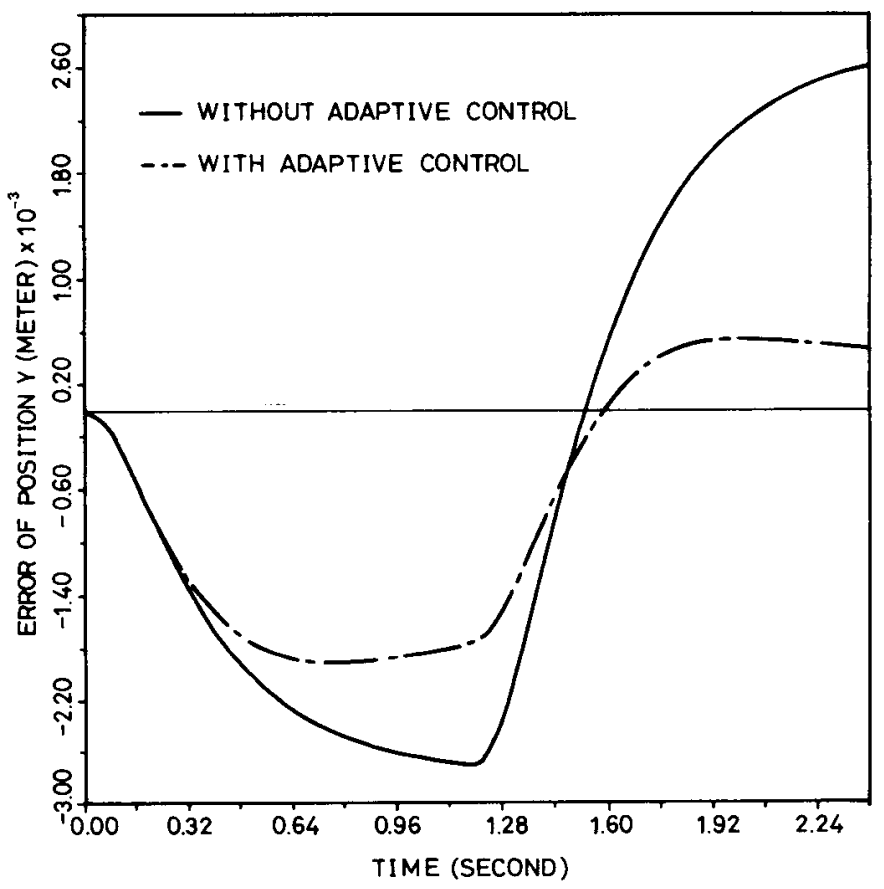

Figure 6. Case 1, position error in $y$-axis. 


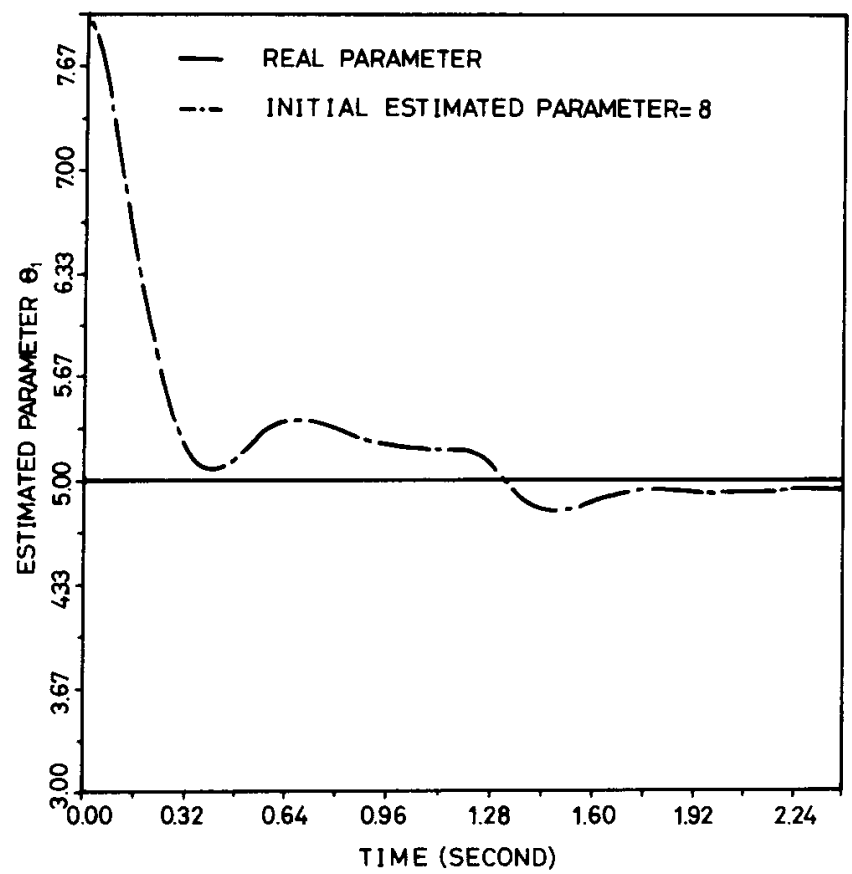

Figure 7. Case 1, convergence of parameter $\boldsymbol{\Theta}_{1}$.

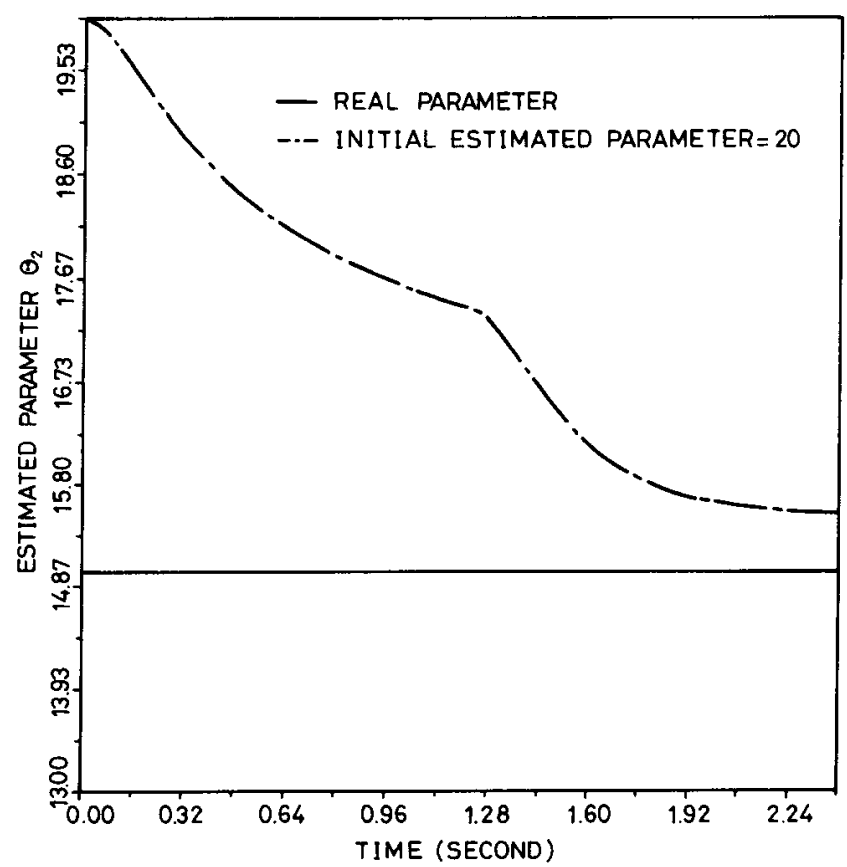

Figure 8. Case 1, convergence of parameter $\boldsymbol{\Theta}_{2}$. 


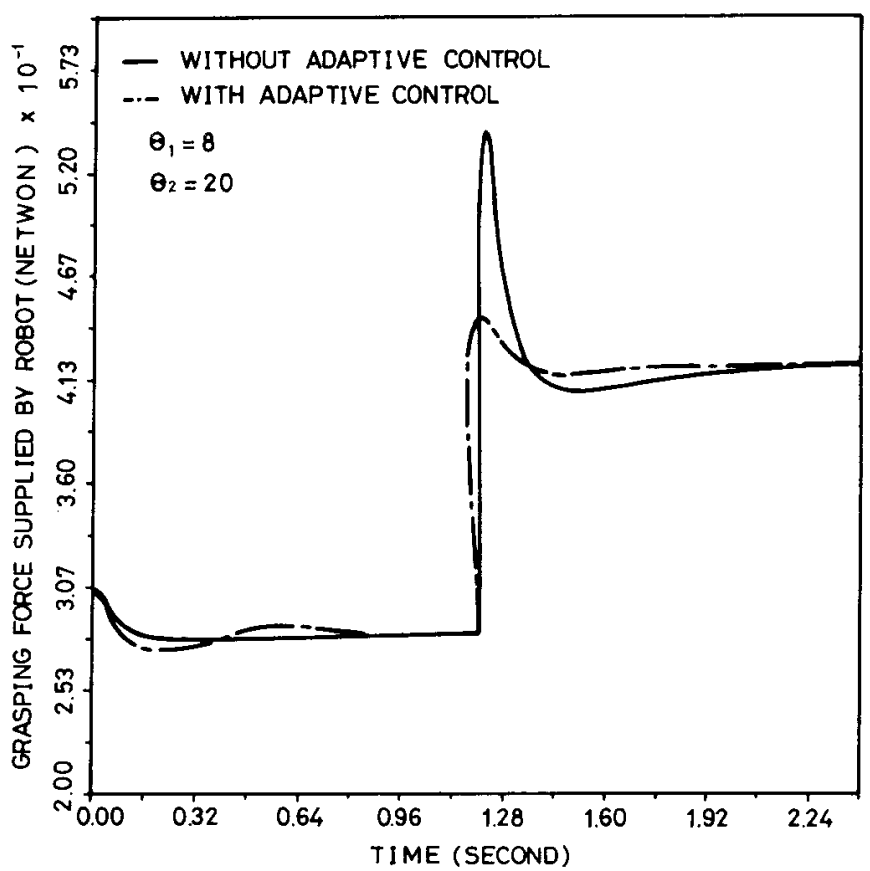

Figure 9. Case 1, the minimum net normal grasping force $\mathbf{N}$.

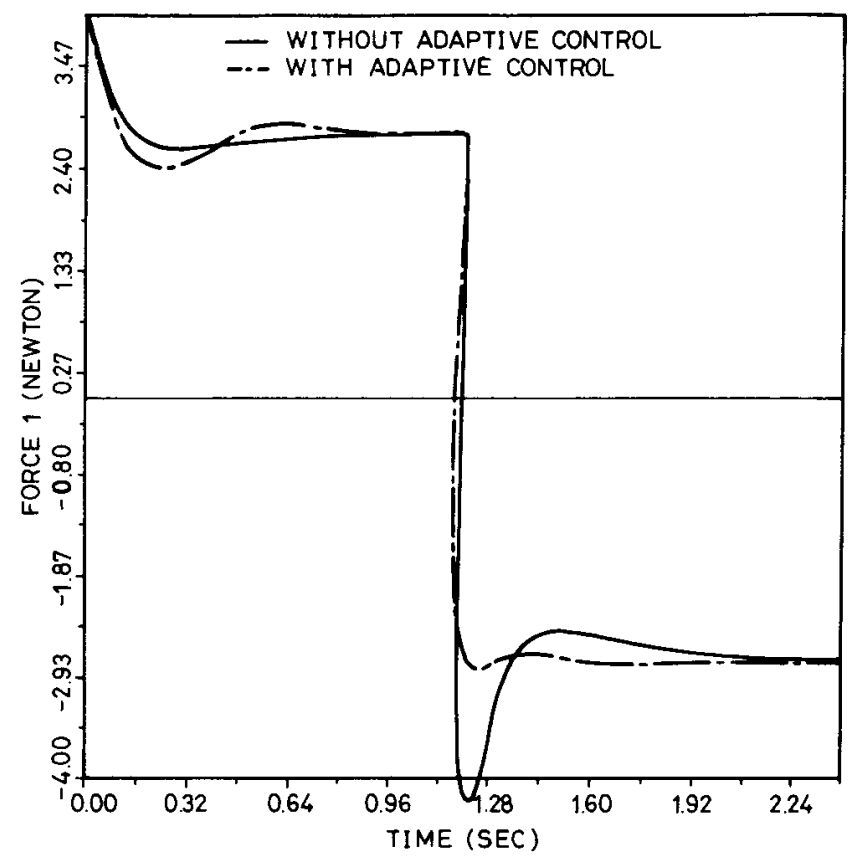

Figure 10. Case 1, joint force of $x$-axis. 


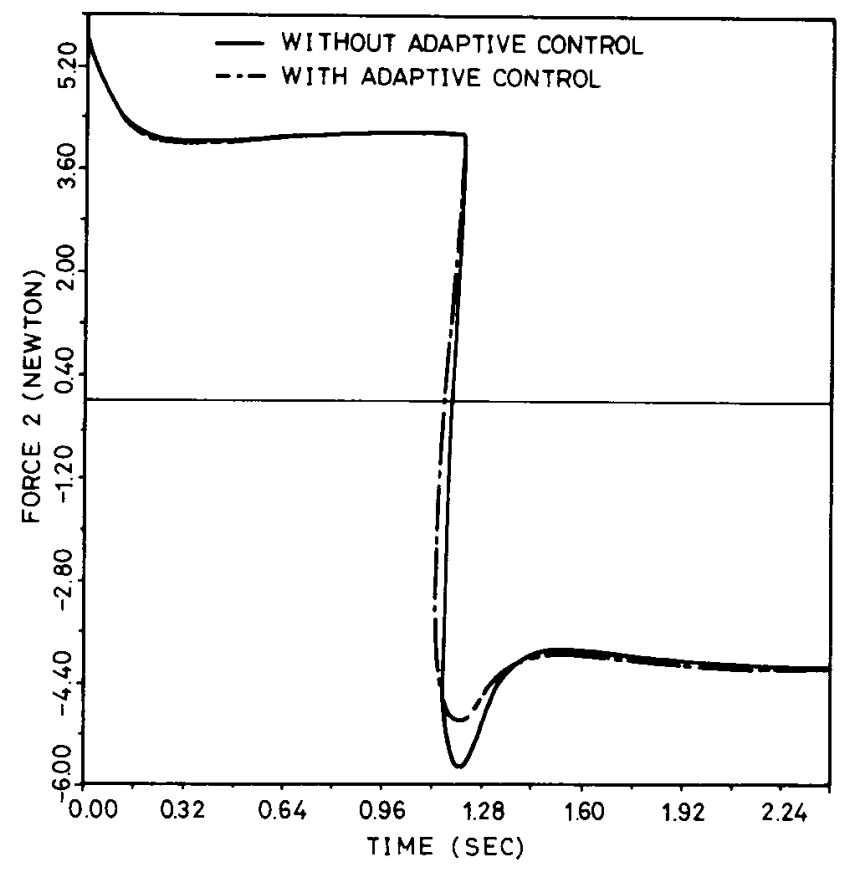

Figure 11. Case 1, joint force of $y$-axis.

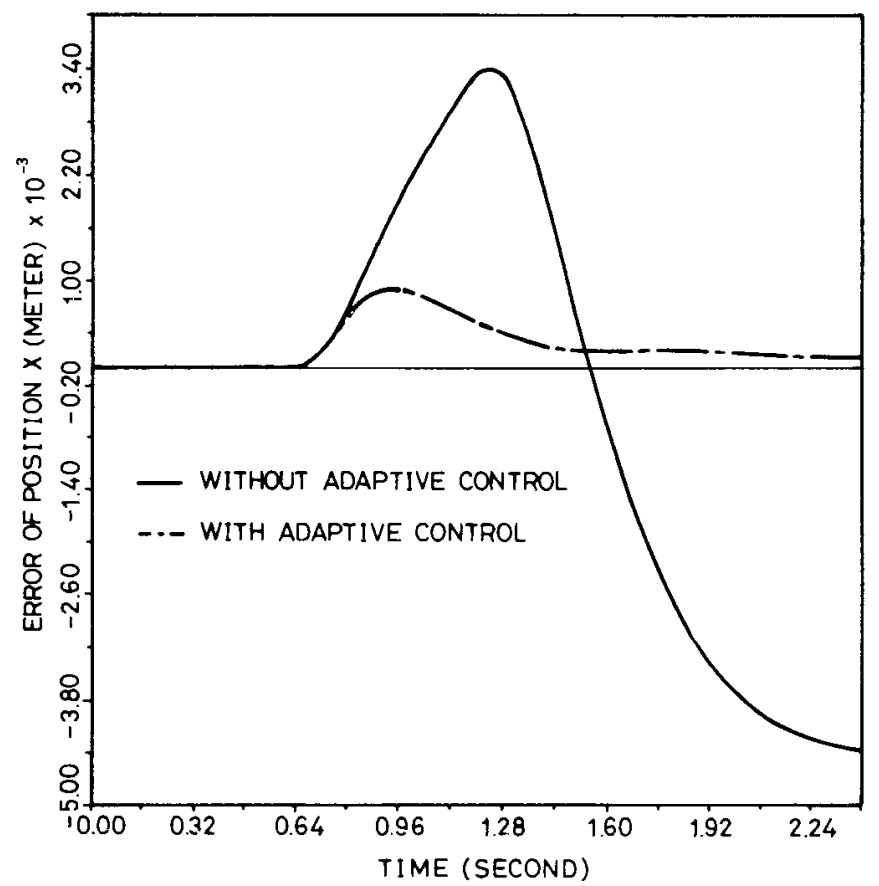

Figure 12. Case 2, position error in $x$-axis. 


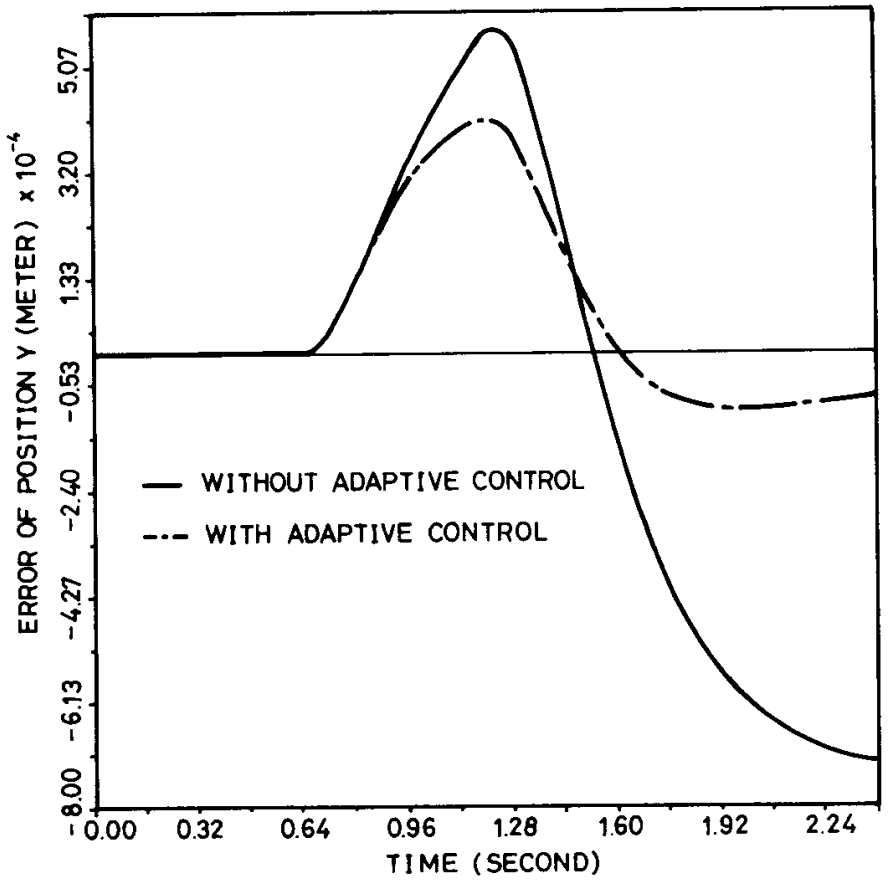

Figure 13. Case 2, position error in y-axis.

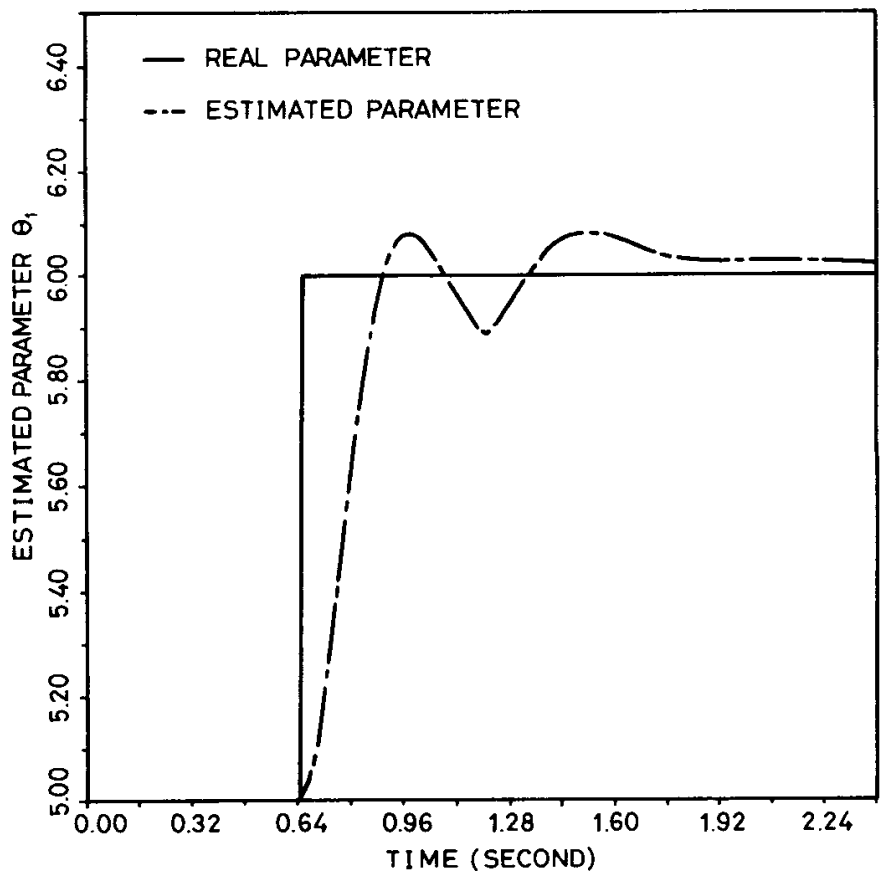

Figure 14. Case 2, convergence of parameter $\boldsymbol{\Theta}_{1}$. 


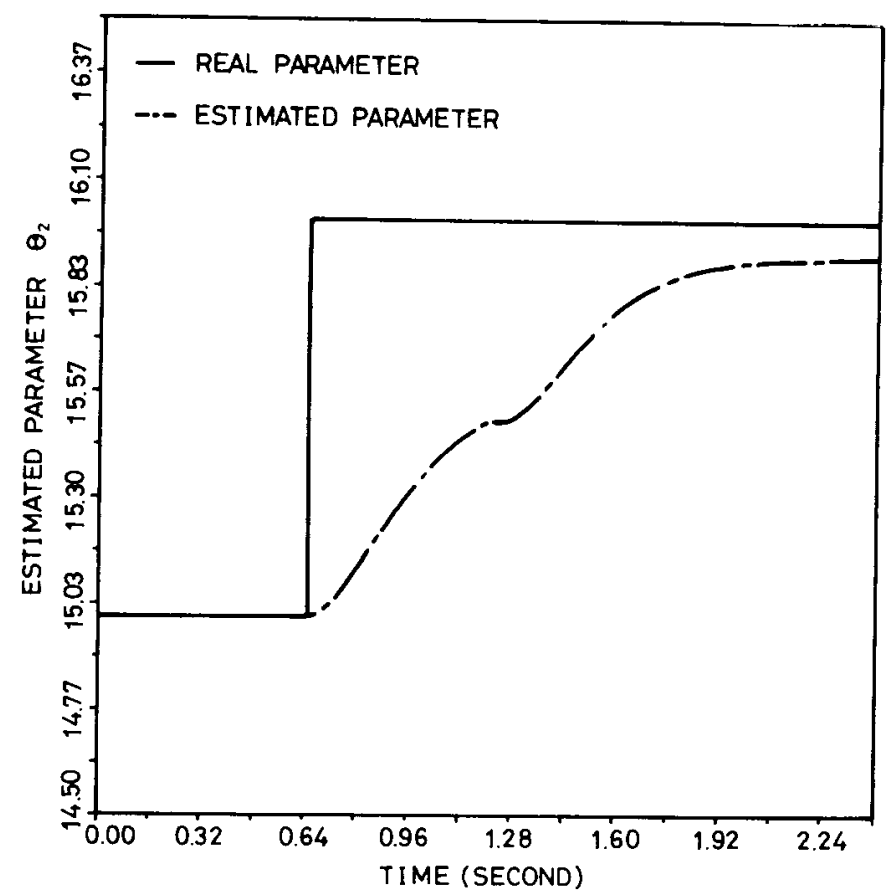

Figure 15. Case 2, convergence of parameter $\boldsymbol{\Theta}_{2}$.

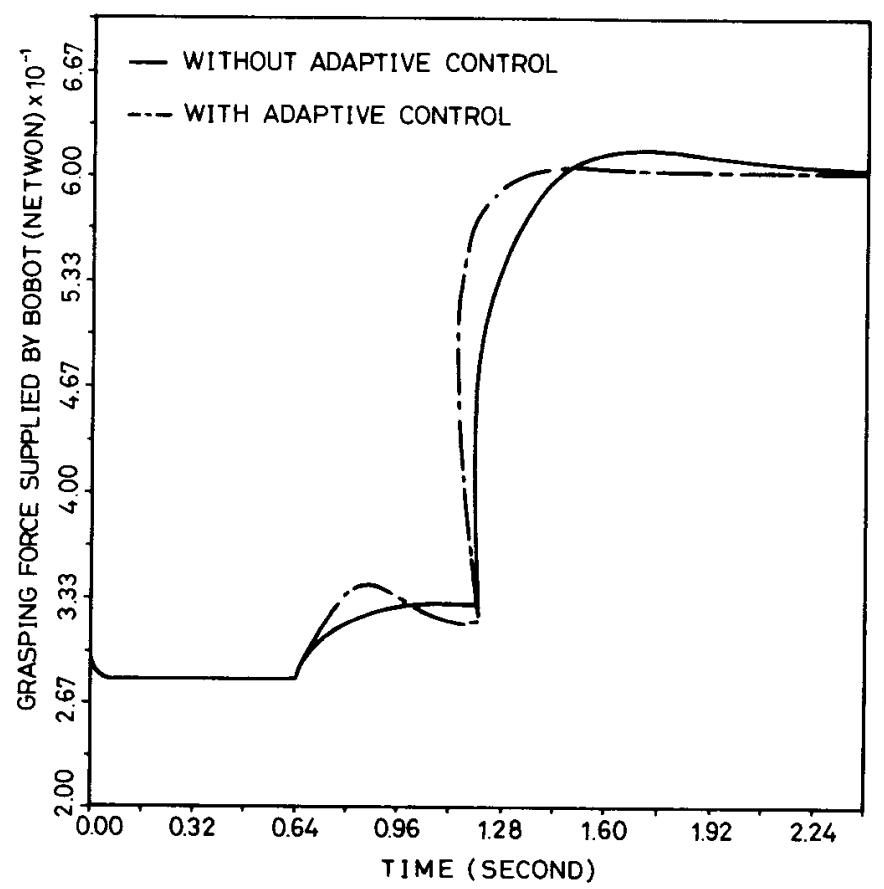

Figure 16. Case 2, minimum net normal grasping for $\mathbf{N}$. 


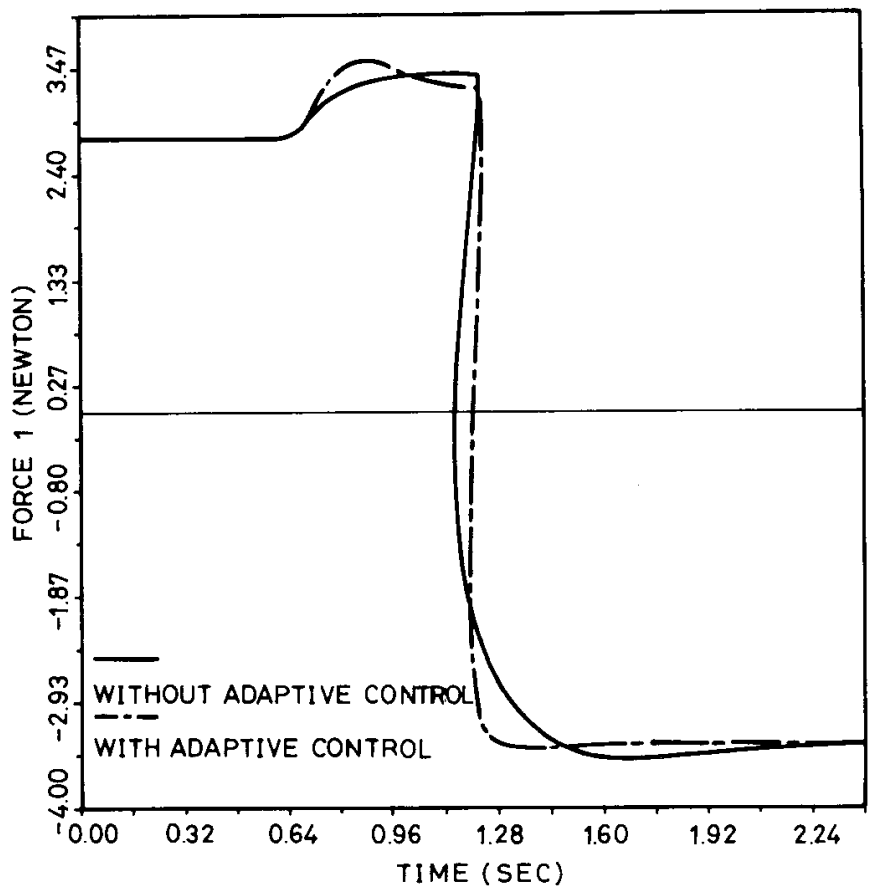

Figure 17. Case 2, joint force of $x$-axis.

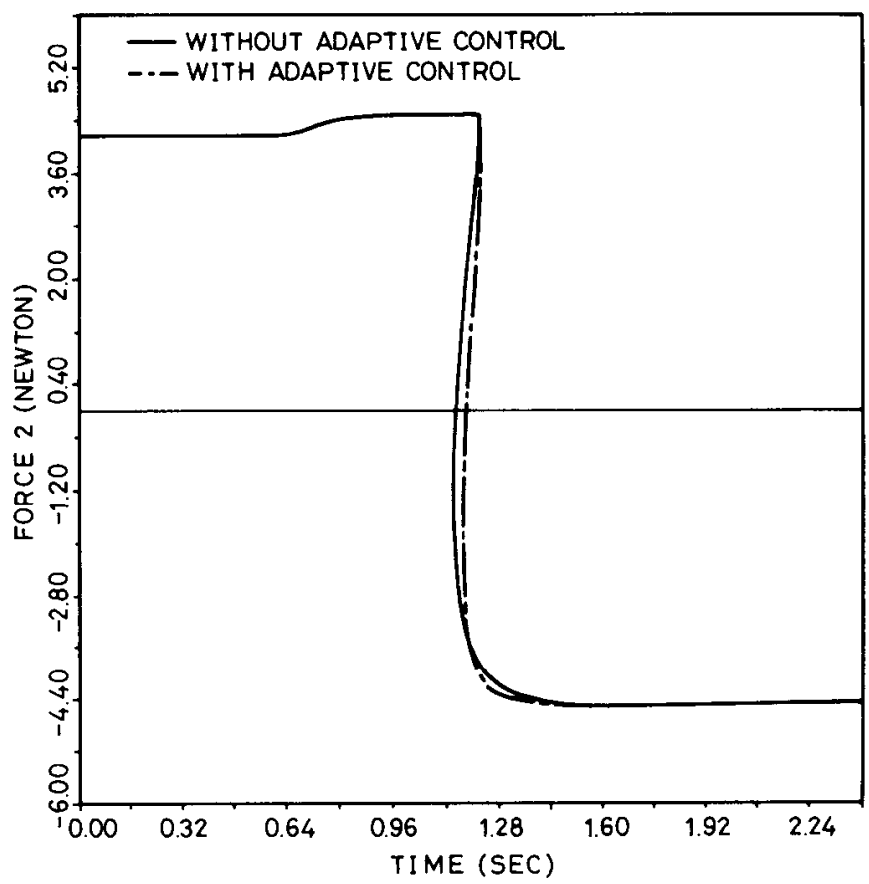

Figure 18. Case 2, joint force of y-axis. 
traditional computed torque controller. It seems that the adaptive controller is able to adapt to the sudden change in the object load while the computer torque controller is unable to do so.

\section{CONCLUSION}

In this article, we developed the complete model for the two-robot system. The model turns out to be a singular system. In coordination control of the tworobot system, the common workspace, the mass, and the inertia of the object should be considered in advance. Otherwise, uncertainties in the dynamic model will result in damage and large position error in the object being handled. The workspace problem can be overcome by the trajectory planner, while the uncertainties in the object dynamics and the robots are alleviated by the proposed adaptive controller. The optimal grasping forces for each robot can be controlled directly or indirectly. It has been shown that the adaptive controller gives a better performance.

The authors thank the reviewers for their unanimous comments. The work of this article was supported in part by the National Science Council, the Republic of China, under Grant NSC-76-0201-E002-05.

\section{References}

1. R. A. Brooks, "Planning collision free motions for pick and place operations," Int. J. of Robotics Research, 2, 19-44 (1983).

2. H. Hoyer, "On-line collision avoidance for industrial robots," Proc. 1st IFAC Symp. Robot Control, 477-485 (1985).

3. T. Lozano-Perez and M. Wesley, "An algorithm for planning collision free paths among polyhedral obstacles," Comm. ACM, 22, 560-570 (1979).

4. F. Dauchey and H. Zapata, "Coordinated control of two cooperative manipulators: The use of a kinematic model," Proc. 15th Int. Symp. Industrial Robots, 641-648 (1985).

5. J. Lim and D. H. Chyung, "On a control scheme for two cooperating robot arms," Proc. of 24th IEEE Conf. on Decision and Control, 1, 334-337 (1985).

6. J. Lim and D. H. Chyung, "Resolved position control for two cooperating robot arms," Robotica, 5, 9-15 (1987).

7. S. Hayati, "Hybrid position/force control of multi-arm cooperating robots," IEEE Int. Conf. on Robotics and Automation, 1, 82-89 (1986).

8. M. Uchiyama, M. N. Iwasawa, and K. Hakomori, "Hybrid position/force control for coordination of a two-arm robot," IEEE Int. Conf. on Robotics and Automation, 2, 1242-1247 (1987).

9. H. P. Huang, "The unified formulation of constrained robot systems," IEEE Int. Conf. on Robotics and Automation, 3, 1590-1592 (1988).

10. Y. F. Zheng and J. Y. S. Luh, "Control of two coorciinated robots in motion," Proc. 24th IEEE Conf. on Decision and Control, 3, 1761-1765 (1985).

11. Y. F. Zheng and J. Y. S. Luh, "Constrained relations between two coordinated industrial robots for motion control," Int. J. of Robotics Research, 6 (1987).

12. T. J. Tarn, A. K. Bejczy, and X. Yun, "Coordinated control of two arm robots," IEEE Int. Conf. on Robotics and Automation, 2, 1193-1198 (1986). 
13. T. J. Tarn, A. K. Bejczy, and X. Yun, "Design of dynamic control of two cooperating robot arms: Closed chain formulation," IEEE Int. Conf. on Robotics and Automation, 1, 7-13 (1987).

14. Y. F. Zheng and J. Y. S. Luh, " Optimal load distribution for two industrial robots, handling a single object," IEEE Int. Conf. on Robotics and Automation, 1, 344349 (1988).

15. T. J. Tarn, A. K. Bejczy, A. Isidori, and Y. L. Chen, "Nonlinear feedback in robot arm control," Proc. of 23rd IEEE Conf. on Decision and Control, 2 (1984).

16. J. J. Craig, P. Hsu, and S. S. Sastry, "Adaptive control of a mechanical manipulator," Int. J. of Robotics Research, 6 (1987).

17. K. S. Fu, R. C. Gonzalez, and C. S. G. Lee, Robotics: Control, Sensing, Vision, and Intelligence, McGraw-Hill, New York, 1987. 